\title{
THE NUB OF AN AUTOMORPHISM OF A TOTALLY DISCONNECTED, LOCALLY COMPACT GROUP
}

\author{
GEORGE A. WILLIS
}

\begin{abstract}
To any automorphism, $\alpha$, of a totally disconnected, locally compact group, $G$, there is associated a compact, $\alpha$-stable subgroup of $G$, here called the $n u b$ of $\alpha$, on which the action of $\alpha$ is topologically transitive. Topologically transitive actions of automorphisms of compact groups have been studied extensively in topological dynamics and results obtained transfer, via the nub, to the study of automorphisms of general locally compact groups.

A new proof that the contraction group of $\alpha$ is dense in the nub is given, but it is seen that the two-sided contraction group need not be dense. It is also shown that each pair $(G, \alpha)$, with $G$ compact and $\alpha$ topologically transitive, is an inverse limit of pairs that have 'finite depth' and that analogues of the Schreier Refinement and Jordan-Hölder Theorems hold for pairs with finite depth.
\end{abstract}

\section{INTRODUCTION}

The automorphisms of a group reveal aspects of its structure, and the present paper extends previous work in 22, 28, 31, 32, on automorphisms of totally disconnected, locally compact groups. Associated with each automorphism, $\alpha$, of the totally disconnected, locally compact group, $G$, there is a compact, $\alpha$-stable subgroup of $G$ here called the $n u b$ of $\alpha$. This paper has two aims: to answer questions from [2] and 32] about the relationship between the nub subgroup and other features of $\alpha$; and to investigate the structure of the nub. Results and examples from topological dynamics found in [25] transfer and assist in meeting these aims.

Concerning the first aim, it is seen in 2 that the contraction group corresponding to $\alpha$ is closed if and only if the nub of $\alpha$ is trivial. Similarly, the second step in the procedure for finding a subgroup tidy for $\alpha$, described in [28, 31, 32, is automatic if the nub is trivial. Section 4 below describes the relationship between the nub of $\alpha$ and the tidying procedure more fully. Several equivalent characterizations of the nub subgroup are also given in that section.

A question left unresolved in 2 and 32 was whether the 'two-sided contraction group', that is, $\left\{x \in G \mid \alpha^{n}(x) \rightarrow 1_{G}\right.$ as $\left.|n| \rightarrow \infty\right\}$ is dense in the nub of $\alpha$. A positive answer to that question would simplify matters in those papers and in Section 4 of this paper. In an attempt to answer the question, the author investigated the structure of the nub of $\alpha$ in more detail and the results of that investigation are reported in Sections 5 and 6 . One of the outcomes is a new proof that the (one-sided) contraction group is dense in the nub: where the original proof in 2] relies on a

1991 Mathematics Subject Classification. Primary: Secondary:

Key words and phrases. group, totally disconnected, automorphism, contraction subgroup, topologically transitive.

Research supported by ARC Discovery Grant DP0984342. 
topological argument, the proof in Section 5 places more reliance on the algebraic structure. Another outcome may be seen in Theorems 6.19 and 6.20, which are a type of Schreier Refinement and Jordan-Hölder Theorem for pairs $(G, \alpha)$, where $G$ is a compact, totally disconnected group and $\alpha \in \operatorname{Aut}(G)$. Unfortunately (perhaps), it turned out that the 'two-sided' contraction group is not always dense in the nub subgroup, as Example 5.11 shows.

The short Section 7 applies the structure theorems for the nub of $\alpha$ obtained in earlier sections to produce an abstract version of a technical result of J. Tits concerning automorphism groups of trees.

As pointed out to me by Klaus Schmidt, much of the work in this paper, including Example 5.11 reproduces results that are already well-known in the topological dynamics literature, see the book 25] for results known prior to 1995 and [6, 19, 21] for more recent work. The main contributions made here appear to be: the link made with the structure theory of non-compact, totally disconnected, locally compact groups; sharper and more algebraic formulations of some results, such as Theorems 6.19 and 6.20 vis-à-vis [25, Proposition 10.2]; and a different perspective and new methods of proof. Where possible, terms from the dynamical systems literature have been adopted. For example, the 'two-sided contraction group' is called the homoclinic subgroup. However, there is one important difference. Automorphisms that are called 'expansive' in the dynamical systems literature are here called 'finite depth': the term 'expansive' conveys a misleading intuition from the point of view of the group structure theory while, on the other hand, 'finite depth' suggests the analogy that motivates Theorems 6.19 and 6.20 .

The next two Sections 2 and 3 review the main concepts from the structure theory of totally disconnected, locally compact groups and from topological dynamics that will be needed in the rest of the paper.

\section{Automorphisms in Structure Theory and Ergodic Theory}

The results in this paper are at the interface between the structure theory of totally disconnected, locally compact groups and the theory of ergodic actions by group automorphisms, and relevant concepts from these theories are recalled here. Key examples that may be used to illustrate ideas in both theories are defined first.

2.1. Product groups and shifts. For each finite group $F$,

$$
F^{\mathbb{Z}}:=\{f: \mathbb{Z} \rightarrow F\}
$$

is the direct product over $\mathbb{Z}$. Equipped with the coordinatewise product and product topology, $F^{\mathbb{Z}}$ is a compact, totally disconnected group. The following particular elements of $\mathbb{Z}$ will be referred to frequently.

Point support: For $f \in F$ and $n \in \mathbb{Z}$, denote by $[f]_{n}$ the element of $F^{\mathbb{Z}}$ such that

$$
[f]_{n}(k)=\left\{\begin{array}{ll}
f & \text { if } k=n \\
1_{F} & \text { if } k \neq n
\end{array} .\right.
$$

Constants: The constant function on $\mathbb{Z}$ with value $f$ will be denoted by $f$ and the subgroup of constant functions, which is isomorphic to $F$, will be denoted by $\underline{F}$. The shift automorphism, $\sigma$, of $F^{\mathbb{Z}}$ is defined by

$$
\sigma(f)(n)=f(n+1)
$$

and the pair $\left(F^{\mathbb{Z}}, \sigma\right)$ will be called the shift. 
In many examples, $F$ is the group $\{\bar{i} \mid i \in\{0,1, \ldots, n-1\}\}$ of integers under addition modulo $n$. This group is isomorphic to the cyclic group of order $n$ and will be denoted $C_{n}$. The ring structure on the integers modulo $n$ will be used on occasion, and $C_{n}$ will then denote this ring (which is a field when $n$ is prime).

Two $\sigma$-stable subgroups of $F^{\mathbb{Z}}$ will be referred to frequently.

The direct sum $\bigoplus_{\mathbb{Z}} F$ will be identified with the subgroup of $F^{\mathbb{Z}}$ consisting of functions with finite support and denoted by $F^{[\mathbb{Z}]}$. Then $F^{[\mathbb{Z}]}$ is generated by the elements $f_{[n]}(f \in F, n \in \mathbb{Z})$ and is a dense subgroup of $F^{\mathbb{Z}}$.

The restricted sum $\left(F^{\mathbb{Z}^{-}}\right) \oplus\left(\bigoplus_{\mathbb{Z} \cup\{0\}} F\right)$ will be identified with the subgroup of $F^{\mathbb{Z}}$ consisting of functions whose support is contained in $(-\infty, n]$ for some $n \in \mathbb{Z}$ and denoted by $F^{\mathbb{Z}<}$. Then $F^{\mathbb{Z}<}$ is a dense subgroup of $F^{\mathbb{Z}}$. It is a non-compact, totally disconnected, locally compact group when equipped with the topology in which all subgroups $F^{(-\infty, n]}$ are compact and open.

It will often be convenient to consider pairs $(G, \alpha)$, where $G$ is a compact group and $\alpha \in \operatorname{Aut}(G)$, as objects in a category in which morphisms, $\phi:(G, \alpha) \rightarrow(H, \beta)$, are group homomorphisms, $\phi: G \rightarrow H$, that intertwine $\alpha$ and $\beta$. It is shown in later sections how general objects in this category may be described in terms of $\operatorname{shifts}\left(F^{\mathbb{Z}}, \sigma\right)$.

2.2. Totally disconnected, locally compact groups and the scale. Each totally disconnected, locally compact group has a base of neighbourhoods of the identity consisting of compact, open subgroups, see [5, 12. Such subgroups will be denoted $V, W, \ldots$ and

$$
\mathcal{B}(G):=\{V \mid V \leq G \text { is compact and open }\} .
$$

Since an open subgroup of a compact group has finite index, any two subgroups in $\mathcal{B}(G)$ are commensurable, that is, their intersection has finite index in both. This allows a positive integer to be associated with each automorphism of $G$, as follows.

Definition 2.1. The scale of the automorphism $\alpha$ is the positive integer

$$
s(\alpha):=\min \{[\alpha(V): \alpha(V) \cap V] \mid V \in \mathcal{B}(G)\} .
$$

A subgroup, $V$, at which the minimum is attained is minimizing for $\alpha$.

When $G$ has a compact, open, normal subgroup all inner automorphisms have scale 1 . The scale can be a useful tool for investigating general totally disconnected, locally compact groups that do not have such a subgroup, see [15, 24, 30].

Applications of the concepts of scale and minimizing subgroup, in [4, 15] for instance, depend on the following structural characterization of minimizing subgroups, which is proved in [28, 31].

Theorem 2.2 (The Structure of Minimizing Subgroups). Let $\alpha$ be an automorphism of $G$. For each $V \in \mathcal{B}(G)$ put

$$
V_{+}=\bigcap_{k \geq 0} \alpha^{k}(V) \text { and } V_{-}=\bigcap_{k \geq 0} \alpha^{-k}(V) .
$$

Then $V$ is minimizing for $\alpha$ if and only if

TA $(\alpha): V=V_{+} V_{-}$and

$\mathbf{T B}(\alpha): V_{++}:=\bigcup_{k>0} \alpha^{k}\left(V_{+}\right)$and $V_{--}:=\bigcup_{k>0} \alpha^{-k}\left(V_{-}\right)$are closed.

If $V$ is minimizing for $\alpha$, then $s(\alpha)=\left[\alpha\left(V_{+}\right): V_{+}\right]$. 
A subgroup satisfying $\mathbf{T A}(\alpha)$ is said to be tidy above for $\alpha$ and one satisfying $\mathbf{T B}(\alpha)$ is tidy below. A subgroup satisfying $\mathbf{T A}(\alpha)$ and $\mathbf{T B}(\alpha)$ is tidy for $\alpha$ so that the theorem may be restated as $V$ is minimizing for $\alpha$ if and only if it is tidy for $\alpha$. The proof of Theorem 2.2 uses a certain procedure for finding subgroups tidy for $\alpha$ that is discussed further in Remark 4.13.

To illustrate these ideas, the only subgroup of $F^{\mathbb{Z}}$ that is minimizing for $\sigma$ is the whole group and $s(\sigma)=1$. On the other hand, all compact, open subgroups $F^{(-\infty, n]}$ of $F^{\mathbb{Z}<}$ are minimizing for the shift automorphism $\sigma$. Putting $V=F^{(-\infty, n]}$, we have $V_{+}=\{1\}, V_{-}=V, s(\sigma)=1$ and $s\left(\sigma^{-1}\right)=|F|$.

The following result is [28, Lemma 1]. It is a key step in the proof of Theorem 2.2 above and will be applied in later sections to automorphisms of compact groups.

Lemma 2.3. Let $G$ be a totally disconnected, locally compact group and let $\alpha$ be an automorphism. Suppose that $V$ is a compact, open subgroup of $G$. Then:

(i) there is $n \in \mathbb{N}$ such that $\bigcap_{k=0}^{n} \alpha^{k}(V)$ is tidy above for $\alpha$, and

(ii) $V_{+}$has finite index in $\alpha\left(V_{+}\right)$.

The following useful criteria for an orbit to be contained in the tidy subgroup $V$ may be found in [31, Lemma 3.2].

Lemma 2.4. Let $V$ be tidy for $\alpha$ and $x \in V$.

(i) If $x \in V_{+}$and $\left\{\alpha^{n}(x)\right\}_{n \in \mathbb{N}}$ is bounded, then $\left\{\alpha^{n}(x)\right\}_{n \in \mathbb{Z}} \leq V$.

(ii) If $\left\{\alpha^{n}(x)\right\}_{n \in \mathbb{Z}}$ is bounded, then $\left\{\alpha^{n}(x)\right\}_{n \in \mathbb{Z}} \leq V$.

2.3. Ergodic, or topologically transitive, actions by automorphisms. The action of a Borel isomorphism, $T$, on a measure space, $(X, \mu)$, is ergodic if every $T$-stable Borel subset of $X$ has either zero or full measure. Beginning with the conjecture of Paul Halmos [10] that a locally compact group having an automorphism that acts ergodically for the Haar measure must be compact, there has been much interest in ergodic theory in the action of single automorphisms on locally compact groups. Halmos' conjecture was proved in [16] in the case of connected groups and in [1 for totally disconnected groups. A short proof of the totally disconnected case that uses the scale of the automorphism and associated ideas is given in 24. An extension of Halmos' conjecture to finitely generated, abelian group of automorphisms acting ergodically is proved in [4.

With the proof of Halmos' conjecture, interest has shifted to ergodic actions of automorphisms of compact groups. The monograph 25] gives a full account of progress up to 1995. Among the numerous publications that have appeared since are [6, 21, 19].

The action of the shift $\sigma$ on the product $F^{\mathbb{Z}}$ is ergodic. The restriction of $\sigma$ to a closed, $\sigma$-stable subgroup, $G$, of $F^{\mathbb{Z}}$ gives rise to a subshift of finite type, see []. Every ergodic pair $(G, \alpha)$, with $G$ compact and totally disconnected, is an inverse limit of subshifts of finite type, see [25. Proposition 2.17] and also Proposition 5.3 below. This key technique in the ergodic theoretic approach that seems to play the corresponding role to that of Lemma 2.3 above in the algebraic approach.

Ergodicity of the pair $(G, \alpha)$ is equivalent to the condition of being topologically transitive, that is, there is $x \in G$ such that the orbit $\left\{\alpha^{n}(x) \mid n \in \mathbb{Z}\right\}$ is dense, 
see [25. Theorem 1.1]. Since the emphasis in this paper is on the algebraic and topological properties of automorphisms, the latter condition will be used here.

\section{Subgroups Associated with an Automorphism}

Several subgroups of $G$ associated with the automorphism $\alpha$ have been studied in the literature. These subgroups and the relationships between them are recalled in this section and a consistent notation for them is introduced.

Definition 3.1. Let $\alpha$ be an automorphism of the totally disconnected, locally compact group $G$.

(i) The contraction group for $\alpha$ is

$$
\operatorname{con}(\alpha):=\left\{x \in G \mid \alpha^{n}(x) \rightarrow 1_{G} \text { as } n \rightarrow \infty\right\} .
$$

(ii) The retraction group for $\alpha$ is

$$
\operatorname{ret}(\alpha):=\bigcap\left\{V_{--} \mid V \in \mathcal{B}(G) \text { is tidy for } \alpha\right\} .
$$

(iii) The bounded contraction group for $\alpha$ is

$$
\operatorname{bco}(\alpha):=\left\{x \in \operatorname{con}(\alpha) \mid\left\{\alpha^{n}(x)\right\}_{n \leq 0} \text { has compact closure }\right\} .
$$

(iv) The two-sided contraction, or homoclinic group for $\alpha$ is

$$
\operatorname{con}_{\leftrightarrow}(\alpha):=\operatorname{con}(\alpha) \cap \operatorname{con}\left(\alpha^{-1}\right) .
$$

(v) The nub group for $\alpha$ is

$$
\operatorname{nub}(\alpha):=\bigcap\{V \mid V \in \mathcal{B}(G) \text { is tidy for } \alpha\} .
$$

The term homoclinic subgroup in (iv) comes from the ergodic theory literature, see [21, and will be adopted in this paper.

The above definitions may be illustrated by considering the shift automorphism on $G=F^{\mathbb{Z}}$, as defined in Subsection 2.1. In this case: $\operatorname{con}(\sigma)=F^{\mathbb{Z}<}=\operatorname{bco}(\sigma)$; $\operatorname{ret}(\sigma)=F^{\mathbb{Z}}=\operatorname{nub}(\sigma)$; and $\operatorname{con}_{\leftrightarrow}(\sigma)=F^{[\mathbb{Z}]}$. Consider also the shift automorphism on $G=F^{\mathbb{Z}<}$. In this case: $\operatorname{con}(\sigma)=F^{\mathbb{Z}<}=\operatorname{ret}(\sigma)$; and $\operatorname{bco}(\sigma)=\operatorname{con}_{\leftrightarrow}(\sigma)=$ $\operatorname{nub}(\alpha)=\{1\}$. Note that, in both of these examples, $\operatorname{con}(\sigma)$ is $\operatorname{dense}$ in $\operatorname{ret}(\sigma)$ and $\operatorname{con}_{\leftrightarrow}(\sigma)$ is dense in $\operatorname{nub}(\sigma)$. It will be seen that the first of these statements holds for every automorphism but that the second does not.

Several of these groups are clearly equal when $G$ is compact.

Proposition 3.2. Let $G$ be a compact, totally disconnected group and $\alpha$ be an automorphism of $G$. Then: $V \leq G$ is tidy for $\alpha$ if and only if it is $\alpha$-stable; $\operatorname{con}(\alpha)=\operatorname{bco}(\alpha) ;$ and $\operatorname{nub}(\alpha)=\operatorname{ret}(\alpha)$.

Remark 3.3. (a) Contraction groups for an automorphism are important in representation theory, 23, 29] and the study of convolution semigroups, 11, 26]. Their role in the structure theory of totally disconnected, locally compact groups is worked out in 2, 7, 13, 14. A rather complete structure theorem for closed contraction groups, that is, when $\operatorname{con}(\alpha)=\operatorname{ret}(\alpha)$, is proved in $[8$.

(b) The contraction group is a dense subgroup of the retraction group, as shown in [2, Theorem 3.26] for metrizable groups and in [13] in the general case. The contraction group is closed, and hence equal to the retraction group, if and only if $\operatorname{nub}(\alpha)=\{1\}$, which occurs if and only if $\operatorname{bco}(\alpha)=\{1\}$, see [2, Theorem 3.32(4)]. 
(c) As a special case, it is shown in [2, Lemma 3.31(2)] that nub $(\alpha)$ is equal to the closure of bco $(\alpha)$ when $G$ is metrizable. Also see Proposition 5.10 in this paper.

(d) Lemma 3.28 in [2] shows that $V_{--} \cap V_{++}=V_{0}:=\bigcap_{n \in \mathbb{Z}} \alpha^{n}(V)$ for every subgroup tidy for $\alpha$. Hence $\operatorname{nub}(\alpha)=\operatorname{ret}(\alpha) \cap \operatorname{ret}\left(\alpha^{-1}\right)$. It is a natural question therefore whether $\operatorname{con}_{\leftrightarrow}(\alpha)$ is dense in nub $(\alpha)$. Proposition 5.10 shows that the closure of $\operatorname{con}_{\leftrightarrow}(\alpha)$ contains the commutator subgroup of nub $(\alpha)$, but Example 5.11 shows that it may be a proper subgroup.

The following additional subgroup associated with $\alpha$ was introduced in 32 .

Definition 3.4. Let $\alpha \in \operatorname{Aut}(G)$ and $V \in \mathcal{B}(G)$. The bounded contraction group relative to $V$ is

$$
\begin{array}{r}
\operatorname{rbco}(\alpha, V):=\left\{x \in G \mid \exists N \text { such that } \alpha^{n}(x) \in V \text { for all } n \geq N\right. \\
\text { and } \left.\left\{\alpha^{n}(x)\right\}_{n \leq 0} \text { has compact closure }\right\} .
\end{array}
$$

This subgroup was used in an argument concerning multiple commuting automorphisms for which it was necessary to characterize property $\mathbf{T B}(\alpha)$ independently of TA $(\alpha)$. This characterization, established in [32, Proposition 2.1], is that any compact, open subgroup containing $\cap\{\overline{\operatorname{rbco}(\alpha, V)} \mid V \in \mathcal{B}(G)\}$ is tidy below 1 .

By definition, $\operatorname{bco}(\alpha)=\bigcap\{\operatorname{rbco}(\alpha, V) \mid V \in \mathcal{B}(G)\}$. Hence

$$
\overline{\mathrm{bco}(\alpha)} \leq \bigcap\{\overline{\operatorname{rbco}(\alpha, V)} \mid V \in \mathcal{B}(G)\} \text {. }
$$

Section 4 returns to the question of characterizing property $\mathbf{T B}(\alpha)$ independently of TA $(\alpha)$ and shows that both groups in (11) are in fact equal to nub $(\alpha)$, thus simplifying some of the arguments in 32 .

\section{The Nub of an Automorphism and Tidy Subgroups}

Compact subgroups of $G$ that are stable under $\alpha$ are the subject of this section, and it is seen that all such subgroups are closely associated with subgroups tidy for $\alpha$. Several characterizations of the nub are derived in addition, and the next few paragraphs explain the significance of these alternative characterizations.

The terms 'tidy above' and 'tidy below' refer to a procedure for determining the scale of the automorphism $\alpha$ and finding a minimizing subgroup. This procedure starts with a given compact, open subgroup, $V$, and modifies it in several steps to produce a minimizing subgroup, see [28, 31]. The aim at each step is to reduce the index $[\alpha(V): \alpha(V) \cap V]$.

In the first step, $n$ is chosen as in Lemma 2.3 to find $V^{\prime}:=\bigcap_{k=0}^{n} \alpha^{k}(V)$ that satisfies TA $(\alpha)$. This step reduces $\alpha(V)$ relative to $\alpha(V) \cap V$ by cutting down $V$, thus making $V$ 'tidy above'.

The next step defines the group $L$ to be the closure of

$$
\mathscr{L}:=\left\{x \in G \mid \alpha^{k}(x) \in V^{\prime} \text { for all but finitely many } k\right\}
$$

and shows that $L$ is compact.

The final step combines $L$ with $V^{\prime}$ to obtain a compact, open subgroup that satisfies $\mathbf{T B}(\alpha)$ as well. In the papers 28, and 31] this is done in two different

\footnotetext{
${ }^{1}$ The subgroup rbco $(\alpha, V)$ is not the same as the contraction group modulo a compact, $\alpha$-stable subgroup $K$ studied in $2,13,14$.
} 
ways that are described in Remark 4.13. This step increases $\alpha(V) \cap V$ relative to $\alpha(V)$ by adding elements to the intersection, thus making $V$ 'tidy below'.

As is apparent from its definition, $L$ depends on the subgroup $V^{\prime}$ produced in the first step. Tidiness below cannot therefore be achieved by this procedure until after tidiness above. The main aim of this section is to show that nub $(\alpha)$, the intersection of all subgroups tidy for $\alpha$, may be used in place of $L$ irrespective of the subgroup $V$ that is given.

4.1. Tidiness and the nub. The next proposition is essentially [2, Lemma 3.31(3)] but, unlike the proof there, does not rely on non-triviality of $\operatorname{con}(\alpha)$.

Proposition 4.1. Let $\alpha$ be an automorphism of $G$. Then a compact, open subgroup that contains $\operatorname{nub}(\alpha)$ and satisfies $\mathbf{T A}(\alpha)$ is tidy for $\alpha$.

Proof. Suppose that $W$ is a compact open subgroup of $G$ with nub $(\alpha) \leq W$ and $W=W_{+} W_{-}$. Denote $W_{+} \cap W_{-}=\bigcap_{n \in \mathbb{Z}} \alpha^{n}(W)$ by $W_{0}$. Applying the tidying procedure to $W$ it suffices, in order to see that $W$ is tidy, to show that the subgroup

$$
\mathscr{L}:=\left\{x \in G \mid \alpha^{k}(x) \in W \text { for all but finitely many } k\right\}
$$

is contained in $W$.

Let $x \in \mathscr{L}$ and observe that there is $k \in \mathbb{Z}$ such that $\alpha^{k}(x) \in W_{+}$. Without loss of generality, suppose that $x \in W_{+}$. Since $W$ is a neighbourhood of $\operatorname{nub}(\alpha)$, there is a subgroup $V \leq W$ that is tidy for $\alpha$. Next, since $W_{0} V$ is a neighbourhood of $W_{0}$ and $\bigcap_{n \geq 0} \alpha^{-n}\left(W_{+}\right)=W_{0}$ is an intersection of compact sets, there is an integer $m$ such that $\alpha^{-m}\left(W_{+}\right) \subset W_{0} V$. Hence, replacing $x$ by $\alpha^{-m}(x)$ for sufficiently large $m$, it may be supposed that $x \in W_{0} V$. Writing $x=x_{0} v$, the orbit $\left\{\alpha^{n}(v)\right\}_{n \in \mathbb{Z}}$ is bounded because $\alpha^{n}(v)=\alpha^{n}\left(x_{0}\right)^{-1} \alpha^{n}(x)$ for each $n$. Since $v$ belongs $V$ which is tidy for $\alpha$, it follows by Lemma 2.4(iii) that $v \in V_{0}:=\bigcap_{n \in \mathbb{Z}} \alpha^{n}(V) \leq W_{0}$. Therefore $x \in W_{0} V_{0}=W_{0}$ and $\alpha^{n}(x) \in W$ for all $n$ as required.

Since the nub is $\alpha$-stable, it follows from Lemma 2.3(1) that

Corollary 4.2. Let $V$ be a compact, open subgroup of $G$ and suppose that $V$ contains $\operatorname{nub}(\alpha)$. Then there is $n \in \mathbb{N}$ such that $\bigcap_{k=0}^{n} \alpha^{k}(V)$ is tidy for $\alpha$.

A close relationship between $\operatorname{nub}(\alpha)$ and condition $\mathbf{T B}(\alpha)$ also follows.

Corollary 4.3. The compact, open subgroup, $V \leq G$ is tidy below for $\alpha \in \operatorname{Aut}(G)$ if and only if $V$ contains $\operatorname{nub}(\alpha)$.

Proof. It must be shown that $V_{++}$is closed if and only if $\operatorname{nub}(\alpha) \leq V$. Choose $n \in \mathbb{N}$ such that $W:=\bigcap_{k=0}^{n} \alpha^{k}(V)$ is tidy above for $\alpha$. Then $W_{+}=V_{+}$and $W_{++}=V_{++}$. Suppose that $\operatorname{nub}(\alpha) \leq V$. Then $\operatorname{nub}(\alpha) \leq W$ and $W_{++}=V_{++}$is closed by Proposition 4.1. Conversely, if $V_{++}$is closed, then so is $W_{++}$and $W$ is tidy for $\alpha$. Then $\operatorname{nub}(\alpha) \leq W \leq V$.

4.2. Tidiness and stability. Tidy subgroups are minimizing for $\alpha$ and, in that sense, come as close as is possible for a compact, open subgroup to be stable under $\alpha$. It is seen next that stable compact subgroups are closely associated with tidy ones.

Proposition 4.4. Let $\alpha \in \operatorname{Aut}(G)$ and suppose that $N$ is a compact, $\alpha$-stable subgroup of $G$ with $\operatorname{nub}(\alpha) \leq N$. Then:

(i) for any open $\mathscr{U} \supset N$ there is an $\alpha$-tidy subgroup $V$ with $N \leq V \subset \mathscr{U}$; 
(ii) for any relatively open, $\alpha$-stable subgroup, $J$, of $N$ there is an $\alpha$-tidy subgroup, $W$, such that $J=W \cap N$.

Proof. (1) Since $\mathscr{U}$ is an open neighbourhood of $N$, there is an open subgroup, $W$, with $N \leq W \subset \mathscr{U}$. Then nub $(\alpha) \leq W$ and so, by Corollary 4.2, there is an integer $n$ such that $\bigcap_{k=0}^{n} \alpha^{k}(W)=: V$ is $\alpha$-tidy. It is clear that $V \subset \mathscr{U}$ and that $N \leq V$ follows from the fact that $N$ is $\alpha$-stable.

(iii) (The claim would follow immediately from (ii) if $\operatorname{nub}(\alpha)$ were contained in $J$. That is not being assumed however.) Since $J$ is open in $N$, there is an open subgroup, $V_{1}$ say, of $G$ such that $V_{1} J \cap N=J$. The smaller open subgroup

$$
V_{2}:=\bigcap\left\{x V_{1} x^{-1} \mid x \in N\right\}
$$

is normalized by $N$, so that the products $V_{2} N$ and $V_{2} J$ are subgroups of $G$. The even smaller subgroup

$$
V_{3}:=V_{2} \cap \alpha^{-1}\left(V_{2}\right)
$$

is normalized by $N$, because $N$ is $\alpha$-stable, and satisfies $\left(V_{3} J\right) \alpha\left(V_{3} J\right) \subset V_{2} J$, because $J$ is $\alpha$-stable. Hence $V_{3} J$ is an open subgroup of $G$ and satisfies

$$
\left(V_{3} J\right) \alpha\left(V_{3} J\right) \cap N \leq V_{2} J \cap N=J .
$$

By part (ii), there is an $\alpha$-tidy subgroup, $V$, between $N$ and $V_{3} N$. Then

$$
W:=V \cap V_{3} J
$$

is a compact, open subgroup of $G$ and $W \cap N=J$. The desired conclusion will be reached once it is shown that $W$ is tidy.

To this end, note first that $V=W N$ because each $v \in V$ is a product $v=x y$ with $x \in V_{3}$ and $y \in N$ where, moreover, $x=v y^{-1}$ belongs to $V_{3} \cap V$, so that in fact $x \in W$. Next, consider

$$
\alpha(W) \cap V=\alpha(W) \cap W N .
$$

If $x$ belongs to this intersection, then $x=w z$ where $w \in W$ and $z \in N$. Then $z=w^{-1} x \in W \alpha(W) \cap N=J$, by (3) and (2). Hence $\alpha(W) \cap V \leq W$ and the map

$$
\alpha(W) /(\alpha(W) \cap W) \rightarrow \alpha(V) /(\alpha(V) \cap V): x(\alpha(W) \cap W) \mapsto x(\alpha(V) \cap V)
$$

is injective. It follows that

$$
[\alpha(W): \alpha(W) \cap W] \leq[\alpha(V): \alpha(V) \cap V] .
$$

However the index on the right is the minimum possible because $V$ is tidy for $\alpha$ and it follows that $W$ is minimizing and therefore is tidy too.

The special case when $N=\operatorname{nub}(\alpha)$ yields [2, Lemma 3.31(2)], but without using density of $\operatorname{con}(\alpha)$ in the proof.

Corollary 4.5. The nub of $\alpha$ has no proper, relatively open $\alpha$-stable subgroups.

The first part of the next result follows from [2, Lemma 3.19] but a short proof is included here.

Proposition 4.6. Let $\alpha \in \operatorname{Aut}(G)$. Then the following hold for any $V \in \mathcal{B}(G)$.

(i) If $V$ is tidy for $\alpha$, then $V \cap K$ is $\alpha$-stable for any compact $\alpha$-stable subgroup $K$ of $G$.

(ii) If $V \cap K$ is $\alpha$-stable for any compact $\alpha$-stable subgroup $K$ of $G$, then $V$ is tidy below for $\alpha$ but need not be tidy above. 
Proof. (ii) Let $x \in V \cap K$. Then $\left\{\alpha^{n}(x)\right\}$ is bounded because it is contained in $K$. Hence $\left\{\alpha^{n}(x)\right\}$ is contained in $V$ by Lemma 2.4

(iii) If $V \cap K$ is $\alpha$-stable for any compact $\alpha$-stable $K$, then in particular $V \cap$ nub $(\alpha)$ is $\alpha$-stable. Since $V \cap \operatorname{nub}(\alpha)$ is an open subgroup of $\operatorname{nub}(\alpha)$, Corollary 4.5 implies that $\operatorname{nub}(\alpha) \leq V$, so that $V$ is tidy below.

The pair $\left(F^{[\mathbb{Z}]}, \sigma\right)$, where $F$ is a finite group and $F^{[\mathbb{Z}]}$ has the discrete topology, has the property that only the trivial subgroup is compact and either $\sigma$-stable or $\sigma$-tidy. Thus every open subgroup satisfies that its intersection with any $\sigma$-stable subgroup is $\sigma$-stable, and yet no non-trivial compact, open subgroup is tidy.

If $K$ is compact, $\alpha$-stable and has no relatively open $\alpha$-stable subgroups, then Proposition 4.6(1i) implies that $K$ is contained in every compact, open subgroup of $G$ that is tidy for $\alpha$, thus yielding the following characterization of $\operatorname{nub}(\alpha)$.

Corollary 4.7. The nub of $\alpha$ is the largest compact, $\alpha$-stable subgroup of $G$ having no relatively open, proper, $\alpha$-stable subgroups.

4.3. Automorphisms acting ergodically. When $G$ is compact, an open subgroup is tidy for $\alpha$ in $\operatorname{Aut}(G)$ if and only if it is $\alpha$-stable. Hence nub $(\alpha)$ is equal to the intersection of all open $\alpha$-stable subgroups of $G$. This intersection is described more precisely in [25. Theorem 1.4] and [14, Theorem 2.6], which apply to general compact groups and not just totally disconnected ones. Specializing to the totally disconnected case, these papers establish the existence of an ordinal $\tau$ and a decreasing family $\left(G_{\rho}\right)_{\rho \in[0, \tau]}$ of closed, $\alpha$-stable subgroups of $G$ such that: $G_{0}=G$; $G_{\rho} / G_{\rho+1}$ is finite for every $\rho \in[0, \tau)$; and $\alpha$ acts ergodically on $G_{\tau}$, which is the largest closed, $\alpha$-stable subgroup of $G$ on which $\alpha$ acts ergodically.

The subgroup $G_{\tau}$ in [14, Theorem 2.6], which is denoted by $G_{\text {erg }}$ in that paper, is equal to $\operatorname{nub}(\alpha)$. Since, as shown in [1, any group on which an automorphism acts ergodically must be compact, Corollary 4.7 and 14 imply the following.

Proposition 4.8. Let $G$ be a totally disconnected, locally compact group and let $\alpha \in \operatorname{Aut}(G)$. Then the action of $\alpha$ on $\operatorname{nub}(\alpha)$ is ergodic and $\operatorname{nub}(\alpha)$ is the largest closed, $\alpha$-stable subgroup of $G$ on which $\alpha$ acts ergodically.

Recall that the the word 'ergodic' in the last proposition could be replaced by 'topologically transitive'. The next result is a restatement of [14, Corollary 2.7(ii)].

Proposition 4.9. Let $G$ be a compact group and $\alpha \in \operatorname{Aut}(G)$. Suppose that $N \triangleleft G$ is a closed, normal $\alpha$-stable subgroup. Then $\operatorname{nub}\left(\left.\alpha\right|^{G / N}\right)=\mathfrak{q}_{N}(\operatorname{nub}(\alpha))$.

Remark 4.10. (a) Example 6.4 from 31 gives a non-compact group $G$ and $\alpha$ in $\operatorname{Aut}(G)$ such that $\operatorname{nub}(\alpha)=\{1\}$ and yet $G$ has a (discrete) normal subgroup $N$ such that $G / N$ is compact and $\operatorname{nub}\left(\left.\alpha\right|^{G / N}\right)=G / N$. Hence Proposition 4.9 may therefore fail if $G$ is not assumed to be compact.

(b) While it is clear that $\mathfrak{q}_{N}\left(\operatorname{con}_{\leftrightarrow}(\alpha)\right) \leq \operatorname{con}_{\leftrightarrow}\left(\left.\alpha\right|^{G / N}\right)$, Example 5.11 shows that equality may fail.

The proofs of the next claims are straightforward.

Proposition 4.11. Let $G$ be a totally disconnected, compact group and $\alpha \in \operatorname{Aut}(G)$. Then $\operatorname{nub}(\alpha), \operatorname{con}(\alpha)$ and $\operatorname{con}_{\leftrightarrow}(\alpha)$ are normal subgroups of $G$.

\footnotetext{
${ }^{2}$ This reference treats only the metrizable case.
} 
4.4. Characterizations of $\operatorname{nub}(\alpha)$ and finding tidy subgroups. The results of this section and elsewhere yield the following characterizations of $\operatorname{nub}(\alpha)$.

Theorem 4.12. Let $G$ be a totally disconnected, locally compact group and $\alpha$ be an automorphism of $G$. Then $\operatorname{nub}(\alpha)$ is the:

(i) intersection of all $\alpha$-tidy subgroups of $G$;

(ii) closure of $\mathrm{bco}(\alpha)$;

(iii) intersection $\bigcap\{\overline{\operatorname{rbco}(\alpha, V)} \mid V \in \mathcal{B}(G)\}$;

(iv) largest compact, $\alpha$-stable subgroup of $G$ having no relatively open $\alpha$-stable subgroups; and

(v) largest compact, $\alpha$-stable subgroup of $G$ on which $\alpha$ acts ergodically.

Proof. The statement in (ii) is the definition of nub( $\alpha$ ), while (iv) and (ㅁ) are respectively Corollary 4.7 and Proposition 4.8 above.

Since, as seen in Equation (11),

$$
\overline{\mathrm{bco}(\alpha)} \leq \bigcap\{\overline{\operatorname{rbco}(\alpha, V)} \mid V \in \mathcal{B}(G)\},
$$

to prove (iii) and (iii), it suffices to show that

$$
\operatorname{nub}(\alpha) \leq \overline{\operatorname{bco}(\alpha)} \text { and } \bigcap\{\overline{\operatorname{rbco}(\alpha, V)} \mid V \in \mathcal{B}(G)\} \leq \operatorname{nub}(\alpha) \text {. }
$$

The first containment holds because, by Corollary 4.7 nub $(\alpha)$ has no compact, open subgroups, and so, by Proposition 5.10, its contraction group is dense.

For the second, following Equation (4) in [32], denote

$$
\bigcap\{\overline{\operatorname{rbco}(\alpha, V)} \mid V \in \mathcal{B}(G)\}=: K \text {. }
$$

Then $K$ is compact by [32, Lemma 2.2] and is $\alpha$-stable because $\mathcal{B}(G)$ is invariant under conjugation. Therefore $K$ is contained in the maximal compact $\alpha$-stable subgroup of $G$ which, by Corollary 4.7, is equal to $\operatorname{nub}(\alpha)$.

Remark 4.13. The characterizations of $\operatorname{nub}(\alpha)$ given in Theorem4.12(ii)-(䜣 admit the following 'algorithm' for finding subgroups tidy for $\alpha$, that is an alternative to those given in 28, 31, 32. Let $V$ be any compact, open subgroup of $G$ and suppose that $\operatorname{nub}(\alpha)$ has been identified by one of these characterizations. Then nub $(\alpha)$ may be embedded in a compact, open subgroup of $G$ obtained from $V$ by either of the two following methods.

The first method defines $V^{\prime \prime}:=\bigcap_{x \in \operatorname{nub}(\alpha)} x V x^{-1}$, which is normalized by nub $(\alpha)$, and is open because there are only finitely many distinct conjugates $x V x^{-1}$ as $x$ ranges over nub $(\alpha)$. Hence $V^{\prime \prime} \operatorname{nub}(\alpha)$ is an open subgroup of $G$ containing nub $(\alpha)$. The second method is given in 31. Define $V^{\prime \prime \prime}:=\{v \in V \mid \operatorname{nub}(\alpha) v \subset V \operatorname{nub}(\alpha)\}$, which is an open subgroup of $G$ and $V^{\prime \prime \prime} \operatorname{nub}(\alpha)=\operatorname{nub}(\alpha) V^{\prime \prime \prime}$, see [31, Lemma 3.3]. Hence $V^{\prime \prime \prime} \operatorname{nub}(\alpha)$ is an open subgroup of $G$ containing $\operatorname{nub}(\alpha)$.

Either method produces a compact, open subgroup, $U$, of $G$ containing nub $(\alpha)$, which therefore satisfies $\mathbf{T B}(\alpha)$, by Corollary 4.3 . There is then a positive integer $n$ such that $\bigcap_{k=0}^{n} \alpha^{k}(U)$ is tidy for $\alpha$, by Corollary 4.2,

\section{Automorphisms of Compact groups}

As seen in the previous section, the intersection of all subgroups tidy for $\alpha$ is the compact subgroup $\operatorname{nub}(\alpha)$ and the restriction of $\alpha$ to $\operatorname{nub}(\alpha)$ is topologically 
transitive. The detailed study of topologically transitive pairs $(G, \alpha)$ begun in this section thus contributes to a more general study of automorphisms of locally compact groups. The approach taken is the same as that in ergodic theory, namely, to express $(G, \alpha)$ as an inverse limit of pairs having a finiteness property called expansiveness in ergodic theory and here called finite depth.

The description of pairs $(G, \alpha)$ as inverse limits is similar to, and is in fact derived from, the theorem that each compact totally disconnected group is an inverse limit of finite groups. Furthering this similarity, each pair $(G, \alpha)$ with finite depth has a depth, which is a positive integer analogous to the order of a finite group. This similarity is behind a more complete description of such pairs in Section 6, where it is shown that a version of the Jordan-Hölder Theorem holds.

\subsection{The finite depth condition.}

Definition 5.1. The pair $(G, \alpha)$ of a compact group and automorphism has finite depth if there is an open subgroup $V \leq G$ such that $\bigcap_{k \in \mathbb{Z}} \alpha^{k}(V)=\{1\}$.

Remark 5.2. (a) In topological dynamics, an automorphism satisfying the finite depth condition is called expansive. That term is not adopted here however because it has misleading connotations. An automorphism of a compact group cannot 'expand' sets: if an open set $\mathscr{U} \subset G$ satisfies $\alpha(\mathscr{U}) \supset \mathscr{U}$, then $\alpha(\mathscr{U})=\mathscr{U}$. The term 'expansive' is also inconsistent [2, where it is said that, if $V=V_{+} V_{-}$is tidy for $\alpha$, then $\alpha$ 'expands' $V_{+}$and 'shrinks' $V_{-}$and that $s(\alpha)$ is the 'expansion factor'. (b) If $V$ is an open subgroup of the compact group $G$ satisfying $\bigcap_{k \in \mathbb{Z}} \alpha^{k}(V)=\{1\}$, then $\bigcap_{x \in G} x V x^{-1}$ is a normal, open subgroup of $G$ satisfying the same condition. Hence the subgroup $V$ in Definition 5.1 may be taken to be normal. This subgroup may also be taken to be tidy above for $\alpha$ by replacing it by $\bigcap_{k=0}^{n} \alpha^{k}(V)$ if necessary, see Lemma 2.3. These additional conditions will usually be assumed to be satisfied.

The following description of compact pairs $(G, \alpha)$ may also be derived from [14, Theorem 5.3] and [18, Theorem 3.6 3 .

Proposition 5.3. Let $G$ be a compact, totally disconnected group and $\alpha \in \operatorname{Aut}(G)$. Then there is an inverse system, $\left\{\left(G_{i}, \alpha_{i}\right), \varphi_{i j}, \mathcal{I}\right\}$ with each pair $\left(G_{i}, \alpha_{i}\right)$ having finite depth such that

$$
(G, \alpha) \cong \lim _{\longleftarrow}\left(G_{i}, \alpha_{i}\right)
$$

Proof. Since $G$ is compact, it has a base, $\left\{V_{i}\right\}_{i \in \mathcal{I}}$, of neighbourhoods of $1_{G}$ consisting of open, normal subgroups. For each $i \in \mathcal{I}$, define $N_{i}=\bigcap_{k \in \mathbb{Z}} \alpha^{k}\left(V_{i}\right)$, $G_{i}=G / N_{i}$ and $\alpha_{i}=\left.\alpha\right|^{G_{i}}$. Then $G_{i}$ is a compact group, $\alpha_{i}$ is a well-defined automorphism, and $\left(G_{i}, \alpha_{i}\right)$ has finite depth. Further define, for $i, j$ with $V_{j} \leq V_{i}$, $\varphi_{i, j}: G_{j} \rightarrow G_{i}$ to be the quotient by $N_{i} / N_{j}$. Then $\left\{G_{i}, \varphi_{i, j}, \mathcal{I}\right\}$ is a projective system of compact groups and $\varphi_{i, j} \circ \alpha_{j}=\alpha_{i} \circ \varphi_{i, j}$. It is routine to check that $(G, \alpha)$ is the claimed inverse limit.

The next result is a special case of [25, Proposition 3.5]. It allows the conditions 'nub $(\alpha)=G$ ' and ' $G$ has no proper open $\alpha$-stable subgroups' to be used interchangeably. The proof given here is based on Lemma 2.3 .

Lemma 5.4. For any compact pair $(G, \alpha)$ with finite depth, $\operatorname{nub}(\alpha)$ is an open subgroup of $G$.

\footnotetext{
${ }^{3}$ In the case of metrizable groups.
} 
Proof. Choose an open subgroup, $V$ of $G$, such that $\bigcap_{k \in \mathbb{Z}} \alpha^{k}(V)=\{1\}$ and $V=$ $V_{+} V_{-}$, see Remark 5.2. Let $W$ be an open $\alpha$-stable subgroup of $G$. It will be shown that both $V_{+}$and $V_{-}$are subgroups of $W$, whence $\operatorname{nub}(\alpha) \geq V$.

Consider first $x \in V_{+}$. Since $\alpha^{-n}(x) \in \bigcap_{k=-n}^{\infty} \alpha^{k}(V)$ for each $n, \alpha^{-n}(x) \rightarrow 1$ as $n \rightarrow \infty$. Hence $\alpha^{-n}(x)$ belongs to $W$ for $n$ sufficiently large. Since $W$ is $\alpha$-stable, $x \in W$ and so $V_{+} \leq W$. That $V_{-} \leq W$ may be shown in a similar way.

The next proposition is the basis for making the notion of 'depth' precise.

Proposition 5.5. Suppose that $(G, \alpha)$ is infinite but has finite depth. Let $V$ be an open subgroup of $G$ with $\bigcap_{k \in \mathbb{Z}} \alpha^{k}(V)=\{1\}$ and $V=V_{+} V_{-}$. Then $\left[\alpha\left(V_{+}\right): V_{+}\right]$is strictly greater than 1 and is independent of the choice of $V$ with these properties.

Proof. Since $G$ is compact, $m(\alpha(V))=m(V)$, where $m$ is Haar measure, and so

$$
\left[\alpha\left(V_{+}\right): V_{+}\right]=\left[V_{-}: \alpha\left(V_{-}\right)\right] .
$$

Hence, if $\left[\alpha\left(V_{+}\right): V_{+}\right]=1$, then $V_{+}$and $V_{-}$are both $\alpha$-stable and, consequently, so is $V$. This can only occur if $V$ is the trivial subgroup and $G$ is finite, a contradiction.

Let $V^{\prime}$ be a second open subgroup, with $\bigcap_{k \in \mathbb{Z}} \alpha^{k}\left(V^{\prime}\right)=\{1\}$ and $V^{\prime}=V_{+}^{\prime} V_{-}^{\prime}$. Then there is an $n \in \mathbb{N}$ such that $\bigcap_{k=-n}^{n} \alpha^{k}\left(V^{\prime}\right) \leq V$. In particular, $\alpha^{-n}\left(V_{+}^{\prime}\right) \leq V_{+}$. Consideration of the inclusions $\alpha\left(V_{+}\right) \geq V_{+} \geq \alpha^{-n}\left(V_{+}^{\prime}\right) \geq \alpha^{-n-1}\left(V_{+}^{\prime}\right)$ yields that

$$
\begin{aligned}
{\left[\alpha\left(V_{+}\right): \alpha^{-n-1}\left(V_{+}^{\prime}\right)\right] } & =\left[\alpha\left(V_{+}\right): V_{+}\right]\left[V_{+}: \alpha^{-n-1}\left(V_{+}^{\prime}\right)\right] \\
& =\left[\alpha\left(V_{+}\right): \alpha^{-n}\left(V_{+}^{\prime}\right)\right]\left[\alpha^{-n}\left(V_{+}^{\prime}\right): \alpha^{-n-1}\left(V_{+}^{\prime}\right)\right] .
\end{aligned}
$$

Since $\alpha$ is an automorphism, $\left[V_{+}: \alpha^{-n-1}\left(V_{+}^{\prime}\right)\right]=\left[\alpha\left(V_{+}\right): \alpha^{-n}\left(V_{+}^{\prime}\right)\right]$ and so

$$
\left[\alpha\left(V_{+}\right): V_{+}\right]=\left[\alpha^{-n}\left(V_{+}^{\prime}\right): \alpha^{-n-1}\left(V_{+}^{\prime}\right)\right]=\left[\alpha\left(V_{+}^{\prime}\right): V_{+}^{\prime}\right] .
$$

Definition 5.6. The depth of $(G, \alpha)$ with finite depth is the index $\left[\alpha\left(V_{+}\right): V_{+}\right]$, where $V$ is any open subgroup of $G$ with $\bigcap_{k \in \mathbb{Z}} \alpha^{k}(V)=\{1\}$ and $V=V_{+} V_{-}$.

The entropy of the action of $\alpha$ on $G$ is the logarithm of the depth, [17, Theorem 2]. The next result, a consequence of Lemma 2.3. will be used in Section 6 to describe the algebraic structure of pairs $(G, \alpha)$ with finite depth.

Lemma 5.7. Suppose that $(G, \alpha)$ has finite depth and let $V$ be an open, normal subgroup of $G$ such that $\bigcap_{k \in \mathbb{Z}} \alpha^{k}(V)=\{1\}$. Then $\alpha^{i}\left(V_{+}\right) \cap \alpha^{j}\left(V_{-}\right)$is a finite normal subgroup of $G$ for every $i, j \in \mathbb{Z}$.

Proof. Since $\alpha^{-1}\left(V_{+}\right) \leq V_{+}$and $\alpha\left(V_{-}\right) \leq V_{-}$, and since $V_{+} \cap V_{-}=\{1\}$, it suffices to consider the case when $j=0$ and $i>0$. For this, note that the quotient map $\alpha^{i}\left(V_{+}\right) \rightarrow \alpha^{i}\left(V_{+}\right) / V_{+}$is injective on $\alpha^{i}\left(V_{+}\right) \cap V_{-}$because $V_{+} \cap V_{-}=\{1\}$. The claim then follows because $\alpha^{i}\left(V_{+}\right) / V_{+}$is finite, see Lemma 2.3

5.2. Density of the contraction group. It is shown in [2, Theorem 3.26] that, if $G$ is a metrizable, locally compact group and $\alpha \in \operatorname{Aut} G$, then $\operatorname{con}(\alpha)$ is dense in $\operatorname{ret}(\alpha)$, and it was shown in [13] that the metrizability restriction could be dispensed with. An alternative proof of the special case when $G$ is compact is given below. Rather than the compactness argument given in [2, the proof uses a more constructive algebraic argument to treat the finite depth case followed by 'approximation by finite depth' using Proposition 5.3 . 
Proposition 5.8. Suppose that the pair $(G, \alpha)$ has finite depth and is topologically transitive. Let $V$ be an open, normal subgroup of $G$ such that $\bigcap_{k \in \mathbb{Z}} \alpha^{k}(V)=\{1\}$ and $V=V_{+} V_{-}$. Then

(i) there is $k \in \mathbb{N}$ such that $\alpha^{k}\left(V_{+}\right) V_{-}=G$; and

(ii) $\bigcup_{n \in \mathbb{N}} \alpha^{n}\left(V_{+}\right) \cap \alpha^{-n}\left(V_{-}\right)$is dense in $G$.

Proof. (ii) The hypothesis that $V$ is normal implies that $V_{+}$and $V_{-}$are normal subgroups of $G$ as well. Hence $\left\{\alpha^{k}\left(V_{+}\right) V_{-}\right\}_{k \in \mathbb{N}}$ is an increasing sequence of subgroups of $G$ that are open because they contain $V$. Since open subgroups have finite index in $G$, this increasing sequence is eventually constant and equal to $\alpha^{k}\left(V_{+}\right) V_{-}$for some $k$. Then

$$
\alpha\left(\alpha^{k}\left(V_{+}\right) V_{-}\right) \subset \alpha^{k+1}\left(V_{+}\right) V_{-}=\alpha^{k}\left(V_{+}\right) V_{-}
$$

and so, since $G$ is compact and $\alpha$ therefore preserves Haar measure, $\alpha^{k}\left(V_{+}\right) V_{-}$ is $\alpha$-stable. The hypothesis that $G$ has no proper open, $\alpha$-stable subgroups then implies that $\alpha^{k}\left(V_{+}\right) V_{-}=G$.

(iii) It will first be shown that $\bigcup_{n \in \mathbb{N}}\left(\alpha^{n}\left(V_{+}\right) \cap V_{-}\right)$is dense in $V_{-}$. For this, consider $x \in V_{-}$. From part (1i), we have that $x \in \alpha^{k+l}\left(V_{+}\right) \alpha^{l}\left(V_{-}\right)$for every $l \in \mathbb{Z}$ whence, for each $l>0$, there is $y \in \alpha^{l}\left(V_{-}\right)$such that $x y \in \alpha^{k+l}\left(V_{+}\right) \cap V_{-}$. Since $\left\{\alpha^{l}\left(V_{-}\right)\right\}_{l>0}$ is a sequence of subgroups that decreases to the trivial subgroup, it follows that $\bigcup_{n \in \mathbb{N}}\left(\alpha^{n}\left(V_{+}\right) \cap V_{-}\right)$is dense in $V_{-}$as claimed. It may be shown by a similar argument that $\bigcup_{n \in \mathbb{N}}\left(\alpha^{k}\left(V_{+}\right) \cap \alpha^{-n}\left(V_{-}\right)\right)$is dense in $\alpha^{k}\left(V_{+}\right)$. This suffices to complete the proof because $G=\alpha^{k}\left(V_{+}\right) V_{-}$.

Since $\alpha^{n}\left(V_{+}\right) \cap \alpha^{-n}\left(V_{-}\right)$is contained in $\operatorname{con}_{\leftrightarrow}(\alpha)$, we have the following.

Corollary 5.9. Let $(G, \alpha)$ be a compact, topologically transitive pair with finite depth. Then $\operatorname{con}_{\leftrightarrow}(\alpha)$ is dense in $G$.

The contraction group is dense even when $(G, \alpha)$ does not have finite depth.

Proposition 5.10. Let $(G, \alpha)$ be a compact, topologically transitive pair. Then

(i) $\operatorname{con}(\alpha)$ is dense in $G$, and

(ii) $\overline{\operatorname{con}_{\leftrightarrow}(\alpha)} \supset[G, G]$.

Proof. (ii) By Proposition [5.3 $G$ is the inverse limit of pairs $\left(G_{i}, \alpha_{i}\right)$ with finite depth where each $G_{i}$ is a quotient, $G / N_{i}$. Then $G_{i}$ inherits from $G$ the property of having no proper open, $\alpha$-stable subgroups and so, by Corollary [5.9, $\operatorname{con}\left(\alpha_{i}\right)$ is dense in $G_{i}$. Hence the contraction subgroup of $\alpha$ modulo $N_{i}$ is dense for each $i$ and it follows, by [13, Theorem 1], that $\operatorname{con}(\alpha)$ is dense in $G$.

(iii) Part (ii) implies that $\operatorname{con}(\alpha)$ and $\operatorname{con}\left(\alpha^{-1}\right)$ are both dense in $G$. Since $\operatorname{con}(\alpha)$ and $\operatorname{con}\left(\alpha^{-1}\right)$ are normal subgroups of $G$,

$$
\left[\operatorname{con}(\alpha), \operatorname{con}\left(\alpha^{-1}\right)\right] \subset \operatorname{con}(\alpha) \cap \operatorname{con}\left(\alpha^{-1}\right)=\operatorname{con}_{\leftrightarrow}(\alpha)
$$

and the claim follows.

It might be thought that $\operatorname{con}_{\leftrightarrow}(\alpha)$ would also be dense in $G$ by the reasoning used to establish Proposition [5.10 (ii). However, the next example, which is the same as [25. Examples 5.6], shows that that is not always the case.

Example 5.11. Let $C_{p}$ be the cyclic group of order $p$ and let $\sigma$ be the shift on $C_{p}^{\mathbb{Z}}$, see Subsection 2.1] The map $\varphi: C_{p}^{\mathbb{Z}} \rightarrow C_{p}^{\mathbb{Z}}$ defined by

$$
\varphi(f)(n)=f(n)-{ }_{p} f(n+1)
$$


is a surjective homomorphism which commutes with $\sigma$ and has kernel the order $p$ subgroup $\underline{C_{p}}$ of constant sequences.

Define an inverse system $\left\{\left(\left(G_{n}, \sigma\right), \varphi_{m, n}\right)\right\}_{n \in \mathbb{N}}$, where $G_{n}=C_{p}^{\mathbb{Z}}$ and $\varphi_{n, n+1}=\varphi$.

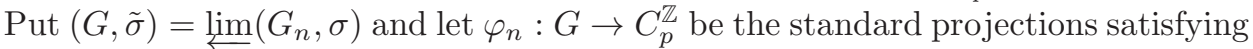
$\varphi_{n}=\varphi_{m} \circ \varphi_{m, n}$. The pair $(G, \tilde{\sigma})$ satisfies the following.

(i) If $V$ is an open subgroup of $G$, then there is $n \in \mathbb{N}$ such that $\operatorname{ker}\left(\varphi_{n}\right) \leq V$, whence $\bigcap_{k \in \mathbb{Z}} \tilde{\sigma}^{k}(V) \neq\{1\}$ and $(G, \tilde{\sigma})$ does not have finite depth.

(ii) Every finite depth quotient of $(G, \tilde{\sigma})$ is isomorphic to $\left(C_{p}^{\mathbb{Z}}, \sigma\right)$ and hence has depth $p$.

(iii) If $f \in \operatorname{con}_{\leftrightarrow}(\tilde{\sigma})$, then $\varphi_{n}(f) \in \operatorname{con}_{\leftrightarrow}(\sigma)$ for every $n$, whence $\varphi_{n}(f)$ has finite support for every $n \in \mathbb{N}$. However, by the definition of $\varphi$ and since $\varphi_{n}=\phi \circ \varphi_{n+1}$, if $f \neq 1$ and

$m=\min \left(\right.$ support of $\left.\varphi_{n+1}(f)\right)$ and $M=\max \left(\right.$ support of $\left.\varphi_{n+1}(f)\right)$,

then $\min \left(\operatorname{support}\right.$ of $\left.\varphi_{n}(f)\right)=m-1$ and $\max \left(\operatorname{support}\right.$ of $\left.\varphi_{n}(f)\right)=M$, that is, the smallest interval supporting $\varphi_{n}(f)$ increases as $n$ decreases. That cannot occur if the support of $\varphi_{n}(f)$ is to be finite and non-empty for every $n$. Hence the support of $\varphi_{n}(f)$ is empty for every $n$ and $f=1$. Therefore $\operatorname{con}_{\leftrightarrow}(\tilde{\sigma})=\{1\}$.

Further to Proposition 5.10 the homoclinic subgroup is not in general a complemented subgroup of $(G, \alpha)$, as the next construction shows.

Example 5.12. Let $\varphi: C_{4}^{\mathbb{Z}} \rightarrow C_{4}^{\mathbb{Z}}$ be the homomorphism defined by

$$
\varphi(x)_{n}=x_{n}-x_{n+1} .
$$

Then $\varphi$ commutes with the shift automorphism, $\sigma$. Hence, setting $H_{k}=C_{4}^{\mathbb{Z}}, \alpha_{k}=\sigma$ and $\varphi_{k, k+1}=\varphi$ for each $k \in \mathbb{N}$ produces an inverse system $\left\{\left(H_{k}, \alpha_{k}\right), \varphi_{k, l}\right\}_{k \leq l \in \mathbb{N}}$ whose inverse limit will be denoted

$$
H:=\lim _{\longleftarrow}\left(C_{4}^{\mathbb{Z}}, \sigma\right)=\left\{\left(x_{k}\right) \in\left(C_{4}^{\mathbb{Z}}\right)^{\mathbb{N}} \mid \phi\left(x_{k+1}\right)=x_{k}\right\} .
$$

The subgroup $2 C_{4}$ of $C_{4}$, which is isomorphic to $C_{2}$, determines a $\sigma$-stable subgroup $\left(2 C_{4}\right)^{\mathbb{Z}} \leq C_{4}^{\mathbb{Z}}$ that is isomorphic to $C_{2}^{\mathbb{Z}}$. Since $2 C_{4}^{\mathbb{Z}}$ is also stable under $\varphi, H$ contains the $\sigma$-stable subgroup

$$
\varliminf_{\longleftarrow}\left(2 C_{4}^{\mathbb{Z}}, \sigma\right)=\left\{\left(x_{k}\right) \in\left(2 C_{4}^{\mathbb{Z}}\right)^{\mathbb{N}} \mid \phi\left(x_{k+1}\right)=x_{k}\right\} \cong \lim _{\longleftarrow}\left(C_{2}^{\mathbb{Z}}, \sigma\right)
$$

and we also have

$$
\lim _{(}\left(C_{4}^{\mathbb{Z}}, \sigma\right) / \lim _{(}\left(2 C_{4}^{\mathbb{Z}}, \sigma\right) \cong \lim _{\longleftarrow}\left(\left(C_{4} / 2 C_{4}\right)^{\mathbb{Z}}, \sigma\right) \cong \lim _{\longleftarrow}\left(C_{2}^{\mathbb{Z}}, \sigma\right) .
$$

Let $\varphi_{1}:\left(x_{k}\right) \mapsto x_{1}$ be the projection of $\lim \left(2 C_{4}^{\mathbb{Z}}, \sigma\right)$ onto its first coordinate. Then the range of $\varphi_{1}$ is isomorphic to $C_{2}^{\mathbb{Z}}$ and $\operatorname{ker} \varphi_{1}$ is a closed, $\sigma$-stable subgroup of $H$. Put $G=H / \operatorname{ker} \varphi_{1}$. Then $\lim \left(2 C_{4}^{\mathbb{Z}}, \sigma\right) / \operatorname{ker} \varphi_{1}$ is a $\sigma$-stable subgroup of $G$ that is isomorphic to $\varphi_{1}\left(2 C_{4}^{\mathbb{Z}}\right) \cong \overleftarrow{C_{2}^{\mathbb{Z}}}$ and the quotient of $G$ by this subgroup is isomorphic to $\varliminf_{2}\left(C_{2}^{\mathbb{Z}}, \sigma\right)$. Therefore

$$
\overline{\operatorname{con}_{\leftrightarrow}(G)}=\lim _{\longleftarrow}\left(2 C_{4}^{\mathbb{Z}}, \sigma\right) / \operatorname{ker} \varphi_{1}
$$

and

$$
G / \overline{\operatorname{con}_{\leftrightarrow}(G)} \cong \lim _{\longleftarrow} C_{2}^{\mathbb{Z}} .
$$


Since both $\overline{\operatorname{con}_{\leftrightarrow}(G)}$ and $C_{2}^{\mathbb{Z}}$ have exponent 2 and $G$ does not, the sequence

$$
\{1\} \longrightarrow \overline{\operatorname{con}_{\leftrightarrow}(G)} \longrightarrow G \longrightarrow \lim _{\longleftarrow} C_{2}^{\mathbb{Z}} \longrightarrow\{1\}
$$

does not split.

The following corollary to Proposition 5.10 is referred to in the next section.

Corollary 5.13. Let $(G, \alpha)$ be topologically transitive and the subgroup $N \leq G$ be finite, normal and $\alpha$-stable. Then $N$ is contained in the centre of $G$.

Proof. Since $N$ is finite and $\alpha$-stable, for any $x \in N$ there is a positive integer $a$ such that $\alpha^{a n}(x)=x$ for all $n \in \mathbb{Z}$. Suppose that $y \in N$ and $h \in \operatorname{con}(\alpha)$. Put $x=h y h^{-1} y^{-1}$ and choose $a$ such that $x=\alpha^{a n}(x)$ and $y=\alpha^{a n}(y)$ for all $n \in \mathbb{Z}$. Then

$$
x=\alpha^{a n}(x)=\alpha^{a n}(h) y \alpha^{a n}\left(h^{-1}\right) y^{-1} \rightarrow 1_{G} \text { as } n \rightarrow \infty .
$$

Hence $y$ is centralized by $\operatorname{con}(\alpha)$ and it follows by Proposition[5.10(ii) that $y$ belongs to the centre of $G$.

\section{A Jordan-Hölder Theorem for Pairs with Finite Depth}

In this section the algebraic structure of compact pairs $(G, \alpha)$ that are topologically transitive and have finite depth is investigated. The main result is Theorem 6.20, which establishes that such pairs have a composition series where the factors are isomorphic to shifts, $\left(F^{\mathbb{Z}}, \sigma\right)$ for some finite simple group $F$, and that the factors are unique up to permutation. This theorem is the direct analogue of the Jordan-Hölder Theorem for finite groups.

Many of the results of this section have counterparts in the ergodic theory literature. It is shown in [17, Proposition 2] that every compact pair $(G, \alpha)$ with finite depth is a subshift of finite type, that is, there is a finite group $F$ such that $(G, \alpha)$ is isomorphic to a closed, $\sigma$-stable subgroup of $\left(F^{\mathbb{Z}}, \sigma\right)$. Indeed, if $V$ is an open, normal subgroup of $G$ with $\bigcap_{n \in \mathbb{Z}} \alpha^{n}(V)=\{1\}$, then $F$ may be taken to be $G / V$. Such subshifts are studied in [17] and a notion of 'block size' introduced. The closest counterpart to that notion in the present paper may be seen in Proposition 5.8 the number $k$ such that $\alpha^{k}\left(V_{+}\right) V_{-}=G$ is related to the block size. The expansive, or finite depth, pair $(G, \alpha)$ is broken down into factors in [17, Theorem 1(ii)] and [25. Proposition 10.2]. These theorems correspond to Proposition 6.3 below, which produces an $\alpha$-stable normal series for $(G, \alpha)$ in which each of the factors is a shift.

6.1. The depth of $(G, \alpha)$. Analogy with the theory of finite groups motivates the results in this section and the depth of the pair $(G, \alpha)$ corresponds to the order of a group under this analogy. The first step is to verify that the depth of pairs on finite depth, see Definition 5.6, behaves as expected under quotients.

Proposition 6.1. Let $H$ be a closed, normal, $\alpha$-stable subgroup of $G$. Then $(G, \alpha)$ has finite depth if and only if both $\left(H,\left.\alpha\right|_{H}\right)$ and $\left(G / H,\left.\alpha\right|^{G / H}\right)$ have finite depth. Moreover,

$$
\operatorname{depth}(G, \alpha)=\operatorname{depth}\left(G / H,\left.\alpha\right|^{G / H}\right) \operatorname{depth}\left(H,\left.\alpha\right|_{H}\right) .
$$

Proof. Suppose that $H$ and $G / H$ have finite depth. Choose open subgroups $W_{1} \leq$ $G / H$ and $W_{2} \leq H$ such that

$$
\bigcap_{k \in \mathbb{Z}}\left(\left.\alpha\right|^{G / H}\right)^{k}\left(W_{1}\right)=\left\{1_{G / H}\right\} \text { and } \bigcap_{k \in \mathbb{Z}} \alpha^{k}\left(W_{2}\right)=\left\{1_{H}\right\} .
$$


Put $V_{1}=\mathfrak{q}_{H}^{-1}\left(W_{1}\right)$, and let $V_{2}$ be an open subgroup of $G$ such that $V_{2} \cap H \leq W_{2}$. Then $V=V_{1} \cap V_{2}$ satisfies $\bigcap_{k \in \mathbb{Z}} \alpha^{k}(V)=\{1\}$ and hence $G$ has finite depth.

For the converse direction, suppose that $G$ has finite depth and let $V$ be an open subgroup such that $\bigcap_{k \in \mathbb{Z}} \alpha^{k}(V)=\left\{1_{G}\right\}$. Then $H$ has finite depth because

$$
\bigcap_{k \in \mathbb{Z}}\left(\left.\alpha\right|_{H}\right)^{k}(V \cap H)=\left\{1_{H}\right\}
$$

That $G / H$ has finite depth cannot be shown by a similar direct argument, see Remark 6.2 below. Instead, it will first be shown that $V$ may be assumed to be normal with $V=V_{+} V_{-}$and to satisfy

$$
\alpha\left(V_{+}\right) \cap V_{-}=\left\{1_{G}\right\} \text { and } \alpha\left(V_{+}\right) V_{-} \cap H=\alpha\left((V \cap H)_{+}\right)(V \cap H)_{-} .
$$

It has already been noted that $V$ may be assumed to be normal. For the other properties note that, since $H$ is $\alpha$-stable, $\alpha(V \cap H)=\alpha(V) \cap H$. Hence, appealing to Lemma 2.3 and replacing $V$ by $\bigcap_{k=0}^{n} \alpha^{k}(V)$ for sufficiently large $n$, it may be supposed that

$$
V=V_{+} V_{-} \text {and } V \cap H=(V \cap H)_{+}(V \cap H)_{-} .
$$

Then, since $V_{+} \cap V_{-}=\left\{1_{G}\right\}$, (6) holds if $V$ is replaced by $\alpha^{-1}(V) \cap V$. All of the subgroups in the above argument are normal because $\alpha$ is an automorphism of $G$.

Assuming that $V$ satisfies (6), it will be shown that

$$
\bigcap_{k=m}^{n} \alpha^{k}(V H) \leq\left(\bigcap_{k=m}^{n} \alpha^{k}(V)\right) H \text { for all } m, n \in \mathbb{Z},
$$

whence $\bigcap_{k \in \mathbb{Z}} \alpha^{k}(V H)=H$ and $\left(G / H,\left.\alpha\right|^{G / H}\right)$ has finite depth as claimed.

To establish (7) when $m=0$ and $n=1$, consider $x$ in $V H \cap \alpha(V H)$. Then

$$
x=v_{1+} v_{1-} y_{1}=\alpha\left(v_{2+}\right) \alpha\left(v_{2-}\right) y_{2} \text {, for some } v_{i \pm} \in V_{ \pm} \text {and } y_{i} \in H \text {. }
$$

Since $\alpha\left(V_{+}\right)$and $V_{-}$are normal subgroups of $G$, (6) implies that $\alpha\left(V_{+}\right) V_{-}$is a direct product, $\alpha\left(V_{+}\right) \times V_{-}$. Hence (8) may be rearranged to yield

$$
\left(v_{1+}^{-1} \alpha\left(v_{2+}\right)\right)\left(v_{1-}^{-1} \alpha\left(v_{2-}\right)\right)=y_{1} y_{2}^{-1},
$$

where the left side belongs to $\alpha\left(V_{+}\right) V_{-}$and the right to $H$. Then (6) implies that there are $w_{ \pm} \in(H \cap V)_{ \pm}$such that

$$
\left(v_{1+}^{-1} \alpha\left(v_{2+}\right)\right)\left(v_{1-}^{-1} \alpha\left(v_{2-}\right)\right)=\alpha\left(w_{+}\right) w_{-} .
$$

Rearranging this equation yields

$$
\alpha\left(w_{+}\right)^{-1}\left(v_{1+}^{-1} \alpha\left(v_{2+}\right)\right)=w_{-}\left(v_{1-}^{-1} \alpha\left(v_{2-}\right)\right)^{-1} .
$$

Since the left side belongs to $\alpha\left(V_{+}\right)$and the right to $V_{-}$and $\alpha\left(V_{+}\right) \cap V_{-}=\left\{1_{G}\right\}$, both sides equal the identity and it follows that

$$
\alpha\left(v_{2+}\right)=v_{1+} \alpha\left(w_{+}\right) \text {, }
$$

which belongs to $V_{+} H$. Substituting into (8) yields that

$$
x=v_{1+} \alpha\left(w_{+}\right) \alpha\left(v_{2-}\right) y_{2} \in V_{+} \alpha\left(V_{-}\right) H=(V \cap \alpha(V)) H
$$

and we have shown that $V H \cap \alpha(V H) \leq(V \cap \alpha(V)) H$. Induction on $n$ and translation by $\alpha^{m}$ imply that (7D) holds for all $m$ and $n$.

\footnotetext{
${ }^{4}$ Tidiness above for $V=V_{+} V_{-}$does not imply the same for $V \cap H$, see [31. Example 6.4], and Lemma 2.3 must be applied to both $V$ and $V \cap H$ separately.
} 
Finally, to establish the formula (55) for the depth, choose $V$ satisfying (6) and recall Definition 5.6 that $\operatorname{depth}(G, \alpha)=\left[\alpha\left(V_{+}\right): V_{+}\right]$. Then

$$
\operatorname{depth}(G, \alpha)=\left[\alpha\left(V_{+}\right): V_{+} H \cap \alpha\left(V_{+}\right)\right]\left[V_{+} H \cap \alpha\left(V_{+}\right): V_{+}\right]
$$

because $V_{+} \leq V_{+} H \cap \alpha\left(V_{+}\right) \leq \alpha\left(V_{+}\right)$. Observe first that, since $V_{+} H$ is a normal subgroup of $G$ and $V_{+} \leq \alpha\left(V_{+}\right)$, the Second Isomorphism Theorem implies that

$$
\alpha\left(V_{+}\right) /\left(V_{+} H \cap \alpha\left(V_{+}\right)\right) \cong \alpha\left(V_{+}\right) H / V_{+} H
$$

and consequently that

$$
\left[\alpha\left(V_{+}\right): V_{+} H \cap \alpha\left(V_{+}\right)\right]=\left[\alpha\left(V_{+}\right) H: V_{+} H\right]=\operatorname{depth}\left(G / H,\left.\alpha\right|^{G / H}\right),
$$

where the last equality holds because $(V H / H)_{+}=\left(V_{+} H\right) / H$ and, as follows from (7), $(V H / H)_{+} \cap(V H / H)_{-}=\{1\}$. Observe second that,

$$
\left(V_{+} H \cap \alpha\left(V_{+}\right)\right) / V_{+} \cong\left(H \cap \alpha\left(V_{+}\right)\right) /\left(H \cap V_{+}\right)
$$

and consequently that

$$
\left[V_{+} H \cap \alpha\left(V_{+}\right): V_{+}\right]=\left[H \cap \alpha\left(V_{+}\right): H \cap V_{+}\right]=\operatorname{depth}\left(H,\left.\alpha\right|_{H}\right) .
$$

Equations (9), (10) and (11) imply (5).

Remark 6.2. Triviality of $\bigcap_{k \in \mathbb{Z}} \alpha^{k}(V)$ does not imply that $\bigcap_{k \in \mathbb{Z}}\left(\left.\alpha\right|^{G / H}\right)^{k}\left(\mathfrak{q}_{H}(V)\right)$ is trivial. Consider $\left(C_{2}^{\mathbb{Z}}, \sigma\right)$ and the subgroups $V=\left\{f \in C_{2}^{\mathbb{Z}} \mid f(0)=\overline{0}\right\}$ and $H$ the set of constant sequences. Then $\bigcap_{k \in \mathbb{Z}} \sigma^{k}(V)=\left\{1_{C_{2}^{\mathbb{Z}}}\right\}$ but $\mathfrak{q}_{H}$ maps $V$ onto $C_{2}^{\mathbb{Z}} / H$.

6.2. An $\alpha$-stable normal series. The first step towards composition series for pairs with finite depth is to show that they have normal series in which the factors are isomorphic to shifts.

Proposition 6.3. Suppose that $(G, \alpha)$ is topologically transitive and has finite depth. Then $G$ has an $\alpha$-stable series

$$
\{1\}=G_{0} \triangleleft G_{1} \triangleleft \cdots \triangleleft G_{r}=G
$$

of closed, normal subgroups, $G_{j}$, and, for each $j \in\{0,1, \ldots, r-1\}$ :

(i) there is a finite group, $F_{j}$, and a surjective homomorphism

$$
\varphi_{j}: F_{j}^{\mathbb{Z}} \rightarrow G_{j+1} / G_{j}
$$

such that $\operatorname{ker}\left(\varphi_{j}\right) \cap F_{j}^{[\mathbb{Z}]}=\{1\}$; and

(ii) $\varphi_{j} \circ \sigma=\alpha_{j} \circ \varphi_{j}$, where $\alpha_{j}=\left.\alpha\right|^{G_{j+1} / G_{j}}$.

Proof. Let $V$ be an open, normal subgroup of $G$ such that $\bigcap_{k \in \mathbb{Z}} \alpha^{k}(V)=\{1\}$. Then $V_{+}$and $V_{-}$are closed, normal subgroups of $G$ and $V_{+} \cap V_{-}=\{1\}$.

Proposition 5.8 (1ii) implies that $\alpha^{l}\left(V_{+}\right) \cap V_{-}$is non-trivial for some $l>0$. Choose $l$ be the smallest such integer and put $F_{0}:=\alpha^{l}\left(V_{+}\right) \cap V_{-}$. Then $F_{0}$ is a finite normal subgroup, by Lemma 5.7 and $\alpha^{l-1}\left(V_{+}\right) \times F_{0} \times \alpha\left(V_{-}\right)$is a direct product of closed, normal subgroups of $G$. Since $\alpha^{n}\left(F_{0}\right)$ is contained in $\alpha\left(V_{-}\right)$when $n>0$ and in $\alpha^{l-1}\left(V_{+}\right)$when $n<0$, it follows that the map $\varphi: F_{0}^{[\mathbb{Z}]} \rightarrow G$ defined by

$$
\varphi(f)=\prod_{n \in \mathbb{Z}} \alpha^{n}(f(n)), \quad\left(f \in F_{0}^{[\mathbb{Z}]}\right),
$$

is injective. Since $\left\{\alpha^{l-n}\left(V_{+}\right) \alpha^{n}\left(V_{-}\right)\right\}_{n \in \mathbb{N}}$ is a base of neighbourhoods of $1_{G}$, and since

$$
\varphi\left(\left\{f \in F_{0}^{[\mathbb{Z}]} \mid f(m)=1 \text { if }-n \leq m \leq n\right\}\right) \leq \alpha^{l-n}\left(V_{+}\right) \alpha^{n}\left(V_{-}\right)
$$


for each $n$, this map extends uniquely to a continuous homomorphism $\varphi_{0}: F_{0}^{\mathbb{Z}} \rightarrow G$ such that $\operatorname{ker}\left(\varphi_{0}\right) \cap F_{0}^{[\mathbb{Z}]}=\{1\}$. By construction, $\alpha \circ \varphi_{0}=\varphi_{0} \circ \sigma$ and $G_{1}:=\varphi_{0}\left(F_{0}^{\mathbb{Z}}\right)$ is a closed, normal $\alpha$-stable subgroup of $G$.

By Proposition 6.1. $\left(G / G_{1},\left.\alpha\right|^{G / G_{1}}\right)$ has finite depth. Hence the argument may be repeated for $\left(G / G_{1},\left.\alpha\right|^{G / G_{1}}\right)$ (provided that it is non-trivial) to produce a closed, normal, $\alpha$-stable subgroup of $G / G_{1}$ that is isomorphic to a quotient of $F_{1}^{\mathbb{Z}}$ for some finite group $F_{1}$. Pulling back to $G$ yields the subgroup, $G_{2}$. Iterating produces subgroups $G_{j}, j=1,2, \ldots$ as required. This iteration terminates at some $r$ with $G_{r}=G$ because Proposition 6.1 and the fact that $\operatorname{depth}\left(G_{j+1} / G_{j}, \alpha_{j}\right)=\left|F_{j}\right|$ imply that the sequence of integers $\left\{\operatorname{depth}\left(G / G_{j},\left.\alpha\right|^{G / G_{j}}\right\}\right.$ strictly decreases so long as $G_{j} \neq G$.

Remark 6.4. The factor in Proposition 6.3 (i) is a quotient of a shift. It will be seen shortly however that, although the homomorphism $\varphi_{j}$ need not be an isomorphism, the factor is in fact isomorphic to a shift.

The calculation in the previous paragraph yields a formula for the depth of $G$.

Corollary 6.5. The depth of the topologically transitive pair $(G, \alpha)$ in Proposition 6.3 is equal to $\prod_{j=0}^{r-1}\left|F_{j}\right|$.

6.3. Normal subgroups of $\left(F^{\mathbb{Z}}, \sigma\right)$. The factors in the $\alpha$-stable normal series (12) are shifts $\left(F^{\mathbb{Z}}, \sigma\right)$, and the next aim is to analyze the structure of these shifts, particularly in the case when $F$ is simple.

Lemma 6.6. Suppose that $H$ is a closed, normal, $\sigma$-invariant subgroup of $F^{\mathbb{Z}}$, where $F$ is a finite simple group. Then exactly one of the following holds:

(i) $H=F^{\mathbb{Z}}$;

(ii) $H=\{1\}$;

(iii) there is $n \in \mathbb{Z}$ such that $H=\left\{h \in F^{\mathbb{Z}} \mid h(j)=1\right.$ for $\left.j \geq n\right\}$;

if $F$ is abelian, $F=C_{p}$ is the cyclic group of order $p$ for some prime $p$, there are $d \in \mathbb{N}$ and $a_{0}, \ldots, a_{d-1} \in C_{p}$ such that

(iv) $H$ is the subgroup of functions satisfying the order d difference equation

$$
H=\left\{h \in F^{\mathbb{Z}} \mid h(r+d)=\sum_{j=0}^{d-1} a_{j} h(r+j) \text { for all } r \in \mathbb{Z}\right\},
$$

in which case $H$ is a finite group with order $p^{d}$; or

(v) there is $n \in \mathbb{Z}$ such that

$$
H=\left\{h \in F^{\mathbb{Z}} \mid h(r+d)=\sum_{j=0}^{d-1} a_{j} h(r+j) \text { for all } r \geq n\right\} .
$$

Proof. Suppose that, for each $n \in \mathbb{Z}$ and $d \geq 0$, there is $h \in H$ with

$$
h(j)=1_{F} \text { for every } j \in\{n, n+1, \ldots, n+d-1\} \text { and } h(n+d) \neq 1_{F} .
$$

Then, since $H$ is normal in $F^{\mathbb{Z}}$ and $F$ is simple, the homomorphism

$$
\pi_{n, d}: H \rightarrow F^{d+1}, \quad h \mapsto(h(n), h(n+1), \ldots, h(n+d))
$$

is surjective for each $n \in \mathbb{Z}$ and $d \geq 0$. Since $H$ is closed, this implies that (ii) holds. Therefore, if (ii) fails, there are $n^{*} \in \mathbb{Z}$ and $d \geq 0$ such that for every $h \in H$

$$
h(j)=1_{F} \text { for } j \in\left\{n^{*}, n^{*}+1, \ldots, n^{*}+d-1\right\} \Longrightarrow h\left(n^{*}+d\right)=1_{F} .
$$


Then $\alpha$-invariance of the subgroup $H$ implies that, for every $h \in H$ and $r \geq n^{*}$,

$$
\text { the values } h(j) \text { for } j \in\{r, r+1, \ldots, r+d-1\} \text { determine } h(r+d) \text {. }
$$

Assume that (1i) fails and consider the case when $F$ is non-abelian. Then, since $H \triangleleft F^{\mathbb{Z}}$, conjugation of $H$ by elements $[f]_{r+d} \in F^{\mathbb{Z}}$ defined by

$$
[f]_{r+d}(j)=\left\{\begin{array}{ll}
f & \text { if } j=r+d \\
1_{F} & \text { if } j \neq r+d
\end{array}, \quad(f \in F),\right.
$$

leads to a contradiction to (13) unless $h(r+d)=1_{F}$ for every $r \geq n^{*}$. Therefore $H$ satisfies either (iii) or (iii) when $F$ is not abelian.

Assume that (ii), (iii) and (iii) fail and let $F=C_{p}$ be abelian. Then (13) and the $\sigma$-invariance of $H$ imply that there are $a_{0}, \ldots, a_{d-1}$ in $C_{p}$ such that every $h \in H$ satisfies

$$
h(r+d)=\sum_{j=0}^{d-1} a_{j} h(r+j) \text { for all } r \geq n^{*} .
$$

(*) Choose d to be the smallest value for which (14) holds.

Should it happen that (14) holds for every $n^{*}$, then either case (iii) or (iv) obtains. Otherwise,

$(* *)$ choose the smallest value of $n^{*}$ for which (14) holds.

Then ( $(\mathbb{\nabla})$ obtains with $n=n^{*}$ : it may be seen immediately that $H$ is contained in the set on the right of the equation and it may be shown that $(*)$ and $(* *)$ imply that $H$ is no smaller than this set.

Lemma 6.7. Let $F$ be a finite group, and suppose that $N$ is a closed, normal $\sigma$ stable subgroup of $F^{\mathbb{Z}}$ such that $N \cap F^{[\mathbb{Z}]}=\{1\}$. Then $N$ is finite and contained in the centre of $F^{\mathbb{Z}}$.

Proof. The finite group $F$ has, by the Jordan-Hölder Theorem, a composition series

$$
\{1\}=F_{0} \triangleleft F_{1} \triangleleft \cdots \triangleleft F_{r-1} \triangleleft F_{r}=F,
$$

and all composition series for $F$ have the same length, $r$. The proof that $N$ is finite will be by induction on $r$. For the base case, note that $N$ is trivial when $r=0$.

Let $F$ have a composition series of length $r>0$ and assume that the claim has been established for groups with shorter composition series. Let $N$ be a closed, normal, $\sigma$-stable subgroup of $F^{\mathbb{Z}}$ such that $N \cap F^{[\mathbb{Z}]}=\{1\}$. Then, considering $F_{r-1}^{\mathbb{Z}}$ to be a subgroup of $F^{\mathbb{Z}}: N \cap F_{r-1}^{\mathbb{Z}}$ is a closed, normal, $\sigma$-stable of $F_{r-1}^{\mathbb{Z}}$ and $N \cap F_{r-1}^{[\mathbb{Z}]}$ is trivial. Hence, by the induction hypothesis,

$$
N \cap F_{r-1}^{\mathbb{Z}} \text { is finite. }
$$

The Second Isomorphism Theorem implies that

$$
N /\left(N \cap F_{r-1}^{\mathbb{Z}}\right) \cong N F_{r-1}^{\mathbb{Z}} / F_{r-1}^{\mathbb{Z}},
$$

which is a closed, normal, $\left.\sigma\right|^{F^{\mathbb{Z}}} / F_{r-1}^{\mathbb{Z}}$-stable subgroup of $F^{\mathbb{Z}} / F_{r-1}^{\mathbb{Z}}$. The map

$$
\varphi: F^{\mathbb{Z}} / F_{r-1}^{\mathbb{Z}} \rightarrow\left(F / F_{r-1}\right)^{\mathbb{Z}}, \quad \varphi\left(f F_{r-1}^{\mathbb{Z}}\right)(j)=f(j) F_{r-1}
$$

is an isomorphism that intertwines $\sigma$. Hence $\varphi\left(N F_{r-1}^{\mathbb{Z}} / F_{r-1}^{\mathbb{Z}}\right)$ is a closed, normal, $\sigma$-stable subgroup of $\left(F / F_{r-1}\right)^{\mathbb{Z}}$, where $F / F_{r-1}$ is a finite simple group because (15) is a composition series. Hence, according to Lemma 6.6 $\varphi\left(N F_{r-1}^{\mathbb{Z}} / F_{r-1}^{\mathbb{Z}}\right)$ falls into one of five cases. Since cases (iii) and ( $\mathbf{\nabla})$ are $\sigma$-invariant but not $\sigma$-stable, 
$\varphi\left(N F_{r-1}^{\mathbb{Z}} / F_{r-1}^{\mathbb{Z}}\right)$ must in fact be either the whole group (case (ii)) or finite (cases (ii) and (iv) ).

It will be shown that

$$
\varphi\left(N F_{r-1}^{\mathbb{Z}} / F_{r-1}^{\mathbb{Z}}\right) \cap\left(F / F_{r-1}\right)^{[\mathbb{Z}]}=\{1\},
$$

which excludes the case when $\varphi\left(N F_{r-1}^{\mathbb{Z}} / F_{r-1}^{\mathbb{Z}}\right)$ is the whole group. To this end, let $f \in N$ be such that $\varphi\left(f F_{r-1}^{\mathbb{Z}}\right)$ belongs to $\left(F / F_{r-1}\right)^{[\mathbb{Z}]}$. Since $N$ is compact, $\left\{\sigma^{k}(f)\right\}_{k \geq 0}$ has an accumulation point, $g$ say, which belongs to $N \cap F_{r-1}^{\mathbb{Z}}$ because

$$
\varphi\left(\sigma^{k}(f) F_{r-1}^{\mathbb{Z}}\right)=\sigma^{k}\left(\varphi\left(f F_{r-1}^{\mathbb{Z}}\right)\right) \rightarrow 1 \text { as } k \rightarrow \infty .
$$

Since $N \cap F_{r-1}^{\mathbb{Z}}$ is a finite $\sigma$-stable subgroup by $(\underline{16}), g$ is periodic. Hence, if the period of $g$ is $p_{1}$, there is an integer $n_{1}$ such that $\left.f\right|_{\left(-\infty, n_{1}\right]}$ is $p_{1}$-periodic. Similarly, there are $p_{2} \geq 1$ and $n_{2} \in \mathbb{Z}$ such that $\left.f\right|_{\left[n_{2}, \infty\right)}$ is $p_{2}$-periodic, and it follows that $f^{-1} \sigma^{p_{1} p_{2}}(f)$ belongs to $N$ and has finite support. Since $N \cap F^{[\mathbb{Z}]}=\{1\}$, we conclude that $f=\beta^{p_{1} p_{2}}(f)$ and hence that $f=g$ and thus belongs to $F_{r-1}^{\mathbb{Z}}$. Therefore, as claimed, $\varphi$ does not map $N F_{r-1}^{\mathbb{Z}} / F_{r-1}^{\mathbb{Z}}$ onto $\left(F / F_{r-1}\right)^{\mathbb{Z}}$ and $N F_{r-1}^{\mathbb{Z}} / F_{r-1}^{\mathbb{Z}}$ is finite. The isomorphism in (17) then implies that $N /\left(N \cap F_{r-1}^{\mathbb{Z}}\right)$ is finite and, combining with (16), it follows that $N$ is finite.

Since $N$ is finite and $\sigma$-stable and $F^{\mathbb{Z}}$ is topologically transitive, Corollary 5.13 shows that it is contained in the centre of $F^{\mathbb{Z}}$.

The factors in the composition series for $(G, \alpha)$ given in Theorem 6.20 below cannot be decomposed any further in the following sense defined in ergodic theory, see 19 for example.

Definition 6.8. The pair $(G, \alpha)$, with $G$ compact and infinite, is irreducible if every proper, closed, normal, $\alpha$-stable subgroup of $G$ is finite.

As might be expected, irreducibility of $(F, \sigma)$ corresponds to simplicity of $F$.

Proposition 6.9. The pair $(G, \alpha)$ is irreducible if and only if it is isomorphic to $\left(F^{\mathbb{Z}}, \sigma\right)$ for some finite simple group $F$.

Proof. Suppose that $G=F^{\mathbb{Z}}$, where $F$ is a finite simple group and let $H$ be a closed, normal $\alpha$-stable subgroup. Consider first the case when $F$ is not abelian. If $H$ is not trivial, then there is $n \in \mathbb{Z}$ and $h \in H$ such that $h(n) \neq 1_{F}$. Then, since $F$ is not abelian, $\left\{\left[h, f^{[n]}\right] \mid f \in F\right\}$ contains a non-identity element and it follows, since $F$ is simple, that $H$ contains $F^{[n]}:=\left\{f^{[n]} \mid f \in F\right\}$. That $H=G$ follows because $H$ is shift-invariant and closed. Consider next the case when $F$ is abelian, that is, $F$ is a cyclic group of order $p$ for some prime $p$. Suppose that $H$ contains a non-identity element, $h$, with finite support. Let $n$ be the minimum of the support of $h$. Then, by adding suitable shifts of powers of $h$, it may be seen that for each $N>0$ there is an element $h^{\prime} \in H$ whose support in the interval $[n-N, n+N]$ is $\{n\}$. Since $H$ is closed and a subgroup, it follows that the elements $\bar{i}^{[n]}\left(\bar{i} \in C_{p}\right)$ belong to $H$. Shift-invariance and closedness of $H$ then imply that $H$ is equal to $G$. Hence, if $H$ is a proper subgroup of $G$, it does not intersect $F^{[\mathbb{Z}]}$ non-trivially and Lemma 6.7 implies that $H$ is finite.

For the converse, assume that $(G, \alpha)$ is irreducible. Then $(G, \alpha)$ has finite depth, by Proposition 5.3. Proposition 6.3 implies that $G$ has an $\alpha$-stable series of the form (12) which, since $(G, \alpha)$ is irreducible, must have $G=G_{1}$. Hence $G=\varphi\left(F^{\mathbb{Z}}\right)$ for some finite group $F$ and $\operatorname{ker}(\varphi) \cap F^{[\mathbb{Z}]}=\{1\}$. By Lemma 6.7 $\operatorname{ker}(\varphi)$ is finite and 
contained in the centre of $F^{\mathbb{Z}}$. Irreducibility of $(G, \alpha)$ then further implies that $\left(F^{\mathbb{Z}}, \sigma\right)$ is irreducible, whence $F$ is simple. Lemma 6.6 then applies to show that either $\operatorname{ker}(\varphi)$ is trivial or $F=C_{p}$ is abelian and

$$
\operatorname{ker}(\varphi)=\left\{h \in F^{\mathbb{Z}} \mid h(r-k)=\sum_{j=0}^{k-1} a_{j} h(r-j) \text { for all } r \in \mathbb{Z}\right\},
$$

for some $a_{0}, \ldots, a_{k-1} \in C_{p}$. The proof is complete in the first case. In the second case, the surjective map $\psi: F^{\mathbb{Z}} \rightarrow F^{\mathbb{Z}}$ defined by

$$
\psi(h)(r)=\sum_{j=0}^{k-1} a_{j} h(r-j)
$$

commutes with $\beta$ and $\operatorname{ker}(\psi)=\operatorname{ker}(\varphi)$. Thus $G \cong F^{\mathbb{Z}} / \operatorname{ker}(\varphi) \cong F^{\mathbb{Z}}$.

The proposition implies the assertion in the next corollary that $\varphi$ does not have a right inverse because the range of any such inverse would a proper, infinite subgroup of $C_{2}^{\mathbb{Z}}$ that is stable under the shift. All other assertions are obvious.

Corollary 6.10. Let $\varphi: C_{2}^{\mathbb{Z}} \rightarrow C_{2}^{\mathbb{Z}}$ be the homomorphism $\varphi(f)_{n}=f_{n}-f_{n+1}$. Then $\varphi$ commutes with the shift automorphism and $C:=\operatorname{ker} \varphi$ is the order 2 subgroup of constant functions in $C_{2}^{\mathbb{Z}}$. The sequence

$$
\{1\} \longrightarrow C \longrightarrow C_{2}^{\mathbb{Z}} \stackrel{\varphi}{\longrightarrow} C_{2}^{\mathbb{Z}} \longrightarrow\{1\}
$$

is exact and does not split.

Remark 6.11. An alternative notion of irreducibility of a topologically transitive pair $(G, \alpha)$ might be that every proper subgroup of $G$ has trivial nub. This weaker definition implies that $(G, \alpha) \cong\left(F^{\mathbb{Z}}, \beta\right)$ in the non-abelian case, but in the abelian case encompasses groups such as those described in Example 5.11.

6.4. The Jordan-Hölder and Schreier Refinement Theorems. Transferring the Schreier Refinement and Jordan-Hölder Theorems from finite groups to pairs $(G, \alpha)$ with finite depth uses a version of the Zassenhaus Lemma, see 20, Lemma 3.3]. The statement of this lemma does not apply without change however because the intersection of topologically transitive $\alpha$-stable subgroups of $G$ need not be topologically transitive, see Example 6.22. The next couple of definitions are required for the modified statement of the lemma.

Definition 6.12. Let $(G, \alpha)$ be a compact group with automorphism $\alpha$. The nub intersection of closed, $\alpha$-stable subgroups $H_{1}$ and $H_{2}$ of $G$ is defined to be

$$
H_{1} \wedge H_{2}=\operatorname{nub}\left(\left.\alpha\right|_{H_{1} \cap H_{2}}\right) \text {. }
$$

Definition 6.13. The pairs $\left(G_{1}, \alpha_{1}\right)$ and $\left(G_{2}, \alpha_{2}\right)$ are co-commensurable if there is a pair $(G, \alpha)$ and surjective homomorphisms $\varphi_{i}: G \rightarrow G_{i}$ such that $\varphi_{i} \circ \alpha_{i}=\alpha \circ \varphi_{i}$ with $\operatorname{ker} \varphi_{i}$ a finite subgroup of $G$, for $i=1,2$. Denote this as $\left(G_{1}, \alpha_{1}\right) \sim_{\mathrm{cc}}\left(G_{2}, \alpha_{2}\right)$.

Remark 6.14. It follows from Proposition 6.9 that two irreducible pairs, $\left(G_{1}, \alpha_{1}\right)$ and $\left(G_{2}, \alpha_{2}\right)$, that are co-commensurable are in fact isomorphic.

Proposition 6.15. $\quad$ (i) Co-commensurability is an equivalence relation.

(ii) The pairs $\left(H_{1}, \alpha_{1}\right)$ and $\left(H_{2}, \alpha_{2}\right)$ are co-commensurable if and only if there is a pair $(L, \gamma)$ and surjective homomorphisms $\psi_{i}: H_{i} \rightarrow L$ with $\gamma \circ \psi_{i}=$ $\psi_{i} \circ \alpha_{i}$ for $i=1,2$, and with $\operatorname{ker}\left(\psi_{i}\right)$ finite. 
Proof. (ii) Reflexivity and symmetry follow immediately from the definition. Suppose that $\left(G_{1}, \alpha_{1}\right),\left(G_{2}, \alpha_{2}\right)$ and $\left(G_{3}, \alpha_{3}\right)$ are pairs with

$$
\left(G_{1}, \alpha_{1}\right) \sim_{\mathrm{cc}}\left(G_{2}, \alpha_{2}\right) \text { and }\left(G_{2}, \alpha_{1}\right) \sim_{\mathrm{cc}}\left(G_{3}, \alpha_{3}\right) .
$$

Transitivity will follow by showing that there is a group $G_{13}$ and surjective homomorphisms $\pi_{12}: G_{13} \rightarrow G_{12}$ and $\pi_{23}: G_{13} \rightarrow G_{23}$ having finite kernels that complete the diagram:

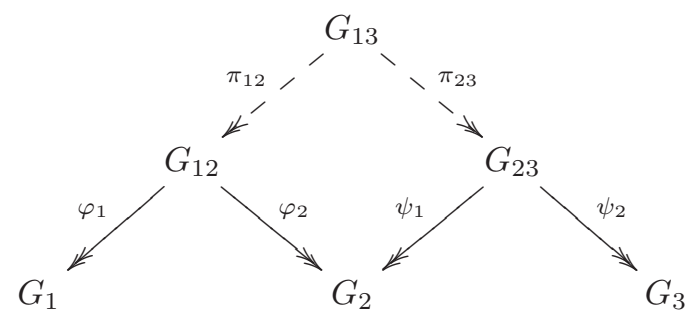

The existence of this group and homomorphisms is proved by applying part (iii) with $H_{1}=G_{12}, H_{2}=G_{23}$ and $L=G_{2}$.

(iii) For the 'if' direction, let $\left(H_{i}, \alpha_{i}\right)$ and $(L, \gamma)$ be pairs such that there are surjective homomorphisms $\psi_{i}: H_{i} \rightarrow L, i=1,2$, that intertwine $\alpha_{i}$ and $\gamma$. Put

$$
H:=\left\{\left(x_{1}, x_{2}\right) \in H_{1} \times H_{2} \mid \psi_{1}\left(x_{1}\right)=\psi_{2}\left(x_{2}\right)\right\}, \alpha=\left.\left(\alpha_{1} \times \alpha_{2}\right)\right|_{H},
$$

and let $\varphi_{i}: H \rightarrow H_{i}$ be the coordinate projections. Then: $\varphi_{1}$ and $\varphi_{2}$ are surjective because $\psi_{1}$ and $\psi_{2}$ are; have $\operatorname{ker} \varphi_{1} \cong \operatorname{ker} \psi_{2}$ and $\operatorname{ker} \varphi_{2} \cong \operatorname{ker} \psi_{1}$; and complete the diagram on the left of Figure 1 Hence $\left(H_{1}, \alpha_{1}\right) \sim_{\mathrm{cc}}\left(H_{2}, \alpha_{2}\right)$.
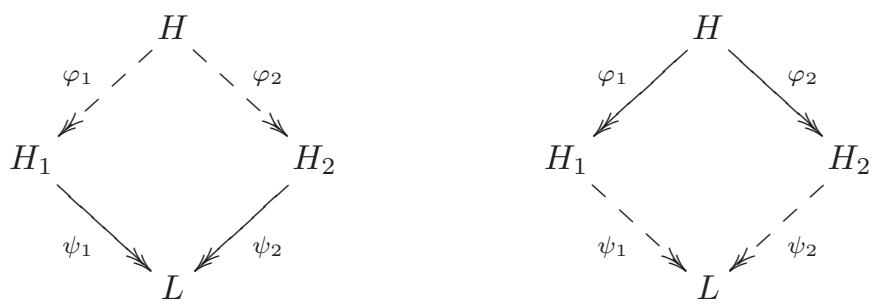

FiguRE 1. An equivalent formulation of co-commensurability

For the 'only if' direction, suppose that $\left(H_{1}, \alpha_{1}\right) \sim_{\mathrm{cc}}\left(H_{2}, \alpha_{2}\right)$ and let $(H, \alpha)$ and $\varphi_{i}: H \rightarrow H_{i}$ are a group and homomorphisms giving effect to their cocommensurability. Put

$$
L:=H /\left(\operatorname{ker}\left(\varphi_{1}\right) \operatorname{ker}\left(\varphi_{2}\right)\right), \quad \gamma=\left.\alpha\right|^{L},
$$

and define $\varphi_{i}:=\rho_{i} \circ \eta_{i}$, where $\eta_{i}: H_{i} \rightarrow H / \operatorname{ker}\left(\varphi_{i}\right)$ is given by the First Isomorphism Theorem, and $\rho_{i}: H / \operatorname{ker}\left(\varphi_{i}\right) \rightarrow H /\left(\operatorname{ker}\left(\varphi_{1}\right) \operatorname{ker}\left(\varphi_{2}\right)\right)$ is the obvious map. Then the pair $(L, \gamma)$ and homomorphisms $\psi_{i}: H_{i} \rightarrow L$ complete the diagram on the right of Figure 1.

One further fact, which is easily verified, is required for the proof of the Zassenhaus Lemma. 
Lemma 6.16. Let $G$ be a compact group and $\alpha \in \operatorname{Aut}(G)$. Suppose that $L$ and $H$ are closed, $\alpha$-stable, topologically transitive subgroups of $G$, and that $L$ is normalized by $H$. Then $L H$ is a closed, $\alpha$-stable, topologically transitive subgroup of $G$.

The Zassenhaus Lemma may now be adapted from [20, Lemma 3.3].

Lemma 6.17. Let $(G, \alpha)$ be a topologically transitive pair with finite depth. Suppose, for $i=1,2$, that $H_{i}$ is a closed, $\alpha$-stable subgroup of $G$ and that $L_{i}$ is a closed, normal, $\alpha$-stable, topologically transitive subgroup of $H_{i}$. Then

$$
\begin{aligned}
& L_{1}\left(H_{1} \wedge L_{2}\right) \text { is normal in } L_{1}\left(H_{1} \wedge H_{2}\right), \\
& \left(L_{1} \wedge H_{2}\right) L_{2} \text { is normal in }\left(H_{1} \wedge H_{2}\right) L_{2},
\end{aligned}
$$

and all groups are $\alpha$-stable and topologically transitive. Denote the automorphisms induced on the respective factor groups by $\alpha_{1}$ and $\alpha_{2}$. Then:

(i) the factor pairs are co-commensurable, that is,

$$
\left(L_{1}\left(H_{1} \wedge H_{2}\right) / L_{1}\left(H_{1} \wedge L_{2}\right), \alpha_{1}\right) \sim_{\mathrm{cc}}\left(\left(H_{1} \wedge H_{2}\right) L_{2} /\left(L_{1} \wedge H_{2}\right) L_{2}, \alpha_{2}\right) \text {; and }
$$

(ii) are topologically transitive.

Proof. Lemma 6.16 implies that all groups are $\alpha$-stable and topologically transitive. The group $L_{1}\left(H_{1} \wedge L_{2}\right)$ is the largest $\alpha$-stable, topologically transitive subgroup of $L_{1}\left(H_{1} \cap L_{2}\right)$. Since, by [20, Lemma 3.3], the latter group is normalized by $L_{1}\left(H_{1} \wedge H_{2}\right)$, which is also $\alpha$-stable, it follows that $L_{1}\left(H_{1} \wedge L_{2}\right)$ is normalized by $L_{1}\left(H_{1} \wedge H_{2}\right)$ as claimed. That $\left(L_{1} \wedge H_{2}\right) L_{2}$ is normal in $\left(H_{1} \wedge H_{2}\right) L_{2}$ may be seen similarly. Proposition 4.9 implies that $L_{1}\left(H_{1} \wedge H_{2}\right) / L_{1}\left(H_{1} \wedge L_{2}\right)$ and $\left(H_{1} \wedge H_{2}\right) L_{2} /\left(L_{1} \wedge H_{2}\right) L_{2}$ are topologically transitive as well, so that (iii) holds.

It remains to prove (i), and this will be deduced from the isomorphism

$$
\frac{L_{1}\left(H_{1} \cap H_{2}\right)}{L_{1}\left(H_{1} \cap L_{2}\right)} \cong \frac{\left(H_{1} \cap H_{2}\right) L_{2}}{\left(L_{1} \cap H_{2}\right) L_{2}}
$$

which is shown in [20, Lemma 3.3].

Since each open $\alpha$-stable subgroup of $L_{1}\left(H_{1} \cap H_{2}\right) / L_{1}\left(H_{1} \cap L_{2}\right)$ pulls back to an open $\alpha$-stable subgroup of $L_{1}\left(H_{1} \cap H_{2}\right)$, it follows that

$$
\operatorname{nub}\left(\frac{L_{1}\left(H_{1} \cap H_{2}\right)}{L_{1}\left(H_{1} \cap L_{2}\right)}\right)=\frac{\left(L_{1}\left(H_{1} \wedge H_{2}\right)\right)\left(L_{1}\left(H_{1} \cap L_{2}\right)\right)}{L_{1}\left(H_{1} \cap L_{2}\right)},
$$

and hence, by the Second Isomorphism Theorem, that

$$
\operatorname{nub}\left(\frac{L_{1}\left(H_{1} \cap H_{2}\right)}{L_{1}\left(H_{1} \cap L_{2}\right)}\right) \cong \frac{L_{1}\left(H_{1} \wedge H_{2}\right)}{L_{1}\left(H_{1} \wedge H_{2}\right) \cap L_{1}\left(H_{1} \cap L_{2}\right)} .
$$

Applying the same argument to the right hand side of (18) yields that

$$
\operatorname{nub}\left(\frac{\left(H_{1} \cap H_{2}\right) L_{2}}{\left.L_{1} \cap H_{2}\right) L_{2}}\right) \cong \frac{\left(H_{1} \wedge H_{2}\right) L_{2}}{\left(H_{1} \wedge H_{2}\right) L_{2} \cap\left(L_{1} \cap H_{2}\right) L_{2}},
$$

whence, from (18),

$$
\frac{L_{1}\left(H_{1} \wedge H_{2}\right)}{L_{1}\left(H_{1} \wedge H_{2}\right) \cap L_{1}\left(H_{1} \cap L_{2}\right)} \cong \frac{\left(H_{1} \wedge H_{2}\right) L_{2}}{\left(H_{1} \wedge H_{2}\right) L_{2} \cap\left(L_{1} \cap H_{2}\right) L_{2}} .
$$

Since $L_{1}\left(H_{1} \wedge L_{2}\right)$ has finite index in $L_{1}\left(H_{1} \cap L_{2}\right)$ and is contained in $L_{1}\left(H_{1} \wedge H_{2}\right)$, the left side of this equation is a quotient of $L_{1}\left(H_{1} \wedge H_{2}\right) / L_{1}\left(H_{1} \wedge L_{2}\right)$ by a finite, normal, $\alpha$-stable subgroup. Similarly, the right side is a quotient of $\left(H_{1} \wedge H_{2}\right) L_{2} /\left(L_{1} \wedge H_{2}\right) L_{2}$ by a finite subgroup. Therefore, by Proposition 6.15,

$$
\frac{L_{1}\left(H_{1} \wedge H_{2}\right)}{L_{1}\left(H_{1} \wedge L_{2}\right)} \sim_{\mathrm{cc}} \frac{\left(H_{1} \wedge H_{2}\right) L_{2}}{\left(L_{1} \wedge H_{2}\right) L_{2}} .
$$


Versions of the Schreier Refinement Theorem, [20, Theorem 3.4], and the JordanHölder Theorem, 20, Theorem 3.5], for topologically transitive pairs with finite depth follow from Lemma 6.17 exactly as they do for finite groups from the Zassenhaus Lemma. Some terminology is needed in order to state these theorems.

Definition 6.18. Let $(G, \alpha)$ be topologically transitive.

(i) An increasing sequence,

$$
\{1\}=G_{0} \leq G_{1} \leq \cdots \leq G_{r-1} \leq G_{r}=G,
$$

of $\alpha$-stable, topologically transitive subgroups of $G$ is a subnormal series for $(G, \alpha)$ if $G_{j-1} \triangleleft G_{j}$ and $\left.\alpha\right|_{G_{j}} ^{G_{j} / G_{j-1}}$ is topologically transitive on $G_{j} G_{j} / G_{j-1}$ for each $j \in\{1,2, \ldots, r\}$. The $j$ th subquotient of the series is the pair $\left(G_{j} / G_{j-1}, \alpha_{j}\right)$, where $\alpha_{j}=\left.\alpha\right|_{G_{j}} ^{G_{j} / G_{j-1}}$.

(ii) Two subnormal series

$$
\begin{aligned}
& \{1\}=G_{0} \triangleleft G_{1} \triangleleft \cdots \triangleleft G_{r-1} \triangleleft G_{r}=G, \\
& \{1\}=H_{0} \triangleleft H_{1} \triangleleft \cdots \triangleleft H_{s-1} \triangleleft H_{s}=G,
\end{aligned}
$$

of $\alpha$-stable, topologically transitive subgroups of $G$ are equivalent if $r=s$ and there is a permutation, $\pi$, of $\{1,2, \ldots, r\}$ such that

$$
\left(G_{j} / G_{j-1}, \alpha_{j}\right) \sim_{\mathrm{cc}}\left(H_{\pi(j)} / H_{\pi(j)-1}, \alpha_{\pi(j)}\right) \text { for each } j .
$$

(iii) A refinement of a subnormal series is subnormal series in which the same subgroups and possibly some others appear.

(iv) A composition series for $(G, \alpha)$ is a subnormal series, as in (ii), for which each subquotient $\left(G_{j} / G_{j-1}, \alpha_{j}\right)$ is an irreducible pair.

Theorem 6.19. Let $(G, \alpha)$ be a topologically transitive pair with finite depth. Then any two subnormal series of $\alpha$-stable, topologically transitive subgroups of $G$ have equivalent refinements.

Theorem 6.20. Let $(G, \alpha)$ be a topologically transitive pair with finite depth. Then $G$ has a composition series. Any two composition series for $(G, \alpha)$ are equivalent.

Remark 6.21. (a) Remark 6.14 implies that the sub-quotients appearing in any two composition series for $(G, \alpha)$ are in fact, up to a permutation, isomorphic.

(b) Suppose that $\left(G_{1}, \alpha_{1}\right)$ and $\left(G_{2}, \alpha_{2}\right)$ are co-commensurable and have finite depth, and let $(G, \alpha)$ and the homomorphisms $\varphi_{i}: G \rightarrow G_{i}$ be as in Definition 6.13. Let

$$
\{1\}=G_{1,0} \triangleleft G_{1,1} \triangleleft \cdots \triangleleft G_{1, r-1} \leq G_{1, r}=G_{1},
$$

be a composition series for $\left(G_{1}, \alpha_{1}\right)$. Then, setting $G_{2, j}$ to be the nub of the restriction of $\alpha_{2}$ to $\varphi_{2} \circ \varphi_{1}^{-1}\left(G_{1, j}\right)$,

$$
\{1\}=G_{2,0} \triangleleft G_{2,1} \triangleleft \cdots \triangleleft G_{2, r-1} \leq G_{2, r}=G_{2},
$$

is a composition series for $\left(G_{2}, \alpha_{2}\right)$ and

$$
\left(G_{1, j} / G_{1, j-1}, \alpha_{1, j}\right) \cong\left(G_{2, j} / G_{2, j-1}, \alpha_{2, j}\right)
$$

for each $j$. Example 6.23 exhibits infinitely many pairs, $\left(G_{n}, \sigma \times \sigma\right), n \in \mathbb{Z}$, having composition series of length $r=2$ and having composition factors $\left(C_{2}^{\mathbb{Z}}, \sigma\right)$ and $\left(C_{3}^{\mathbb{Z}}, \sigma\right)$ but which are not isomorphic because they are semidirect products with different actions of $\left(C_{2}^{\mathbb{Z}}, \sigma\right)$ by automorphisms on $\left(C_{3}^{\mathbb{Z}}, \sigma\right)$. 
Example 6.22. The group $G=C_{2}^{\mathbb{Z}} \times C_{2}^{\mathbb{Z}}$ with $\alpha=\sigma \times \sigma$ is clearly the internal direct product of $\alpha$-stable subgroups

$$
H_{1}=C_{2}^{\mathbb{Z}} \times\{1\} \text { and } H_{2}=\{1\} \times C_{2}^{\mathbb{Z}} .
$$

However $(G, \alpha)$ is a product of $\alpha$-stable subgroups in many other ways.

Define $H_{\varphi}$ to be the subgroup

$$
H_{\varphi}=\left\{(\varphi(x), x) \in G \mid x \in C_{2}^{\mathbb{Z}}\right\},
$$

where $\varphi: C_{2}^{\mathbb{Z}} \rightarrow C_{2}^{\mathbb{Z}}$ is a surjective homomorphism. For example, $\varphi$ might be the homomorphism defined in Corollary 6.10. Then $H_{\varphi}$ is a closed $\alpha$-stable subgroup of $G$ that isomorphic to $C_{2}^{\mathbb{Z}}$.

If $(x, y) \in H_{1} \cap H_{\varphi}$, then $y=1$ because $(x, y) \in H_{1}$, which forces $x=\varphi(y)=1$ because $(x, y) \in H_{\varphi}$. Hence $H_{1} \cap H_{\varphi}=\{1\}$ and $G=H_{1} \times H_{\varphi}$. On the other hand, $(0, y) \in H_{2} \cap H_{\varphi}$ if and only if $y \in \operatorname{ker} \varphi$. Hence, although $G=H_{2} H_{\varphi}$, it is not the direct product of $\mathrm{H}_{2}$ and $\mathrm{H}_{\varphi}$.

That $H_{2} \cap H_{\varphi}=\{1\} \times \operatorname{ker} \varphi$ shows that the intersection of topologically transitive $\alpha$-stable subgroups need not be topologically transitive. Note however that $\operatorname{ker} \varphi$ is a finite subgroup of $C_{2}^{\mathbb{Z}}$ and so $H_{2} \wedge H_{\varphi}=\{1\}$.

Example 6.23. The symmetric group $S_{3}$ is isomorphic to the semidirect product $C_{3} \rtimes_{\chi} C_{2}$ determined by the homomorphism $\chi: C_{2} \rightarrow \operatorname{Aut}\left(C_{3}\right)$ that sends $\overline{1} \in C_{2}$ to the automorphism $\overline{1} \mapsto \overline{2}$ of $C_{3}$. The group $S_{3}^{\mathbb{Z}}$ is consequently isomorphic to the semidirect product $C_{3}^{\mathbb{Z}} \rtimes_{\chi_{0}} C_{2}^{\mathbb{Z}}$ determined by the homomorphism $\chi_{0}: C_{2}^{\mathbb{Z}} \rightarrow$ $\operatorname{Aut}\left(C_{3}^{\mathbb{Z}}\right)$ that sends $[\overline{1}]_{s} \in C_{2}^{\mathbb{Z}}$ to the automorphism such that

$$
\chi_{0}\left([\overline{1}]_{s}\right)(f)(r)=\left\{\begin{array}{ll}
\chi(f(r)), & \text { if } r=s \\
f(r), & \text { otherwise }
\end{array}, \quad\left(f \in C_{3}^{\mathbb{Z}}\right) .\right.
$$

Let $\left(\lim _{\longleftarrow} C_{2}^{\mathbb{Z}}, \tilde{\sigma}\right)$ be the pair defined in Example 5.11 and $\varphi_{1}: \lim _{2} C_{2}^{\mathbb{Z}} \rightarrow C_{2}^{\mathbb{Z}}$ be the homomorphism also defined there. Then $\chi_{0} \circ \varphi_{1}: \lim _{\leftarrow} C_{2}^{\mathbb{Z}} \rightarrow \operatorname{Aut}\left(C_{3}^{\mathbb{Z}}\right)$ is a homomorphism that commutes with the automorphisms $\sigma \in \operatorname{Aut}\left(C_{3}^{\mathbb{Z}}\right)$ and $\tilde{\sigma} \in \operatorname{Aut}\left(\lim _{\longleftarrow} C_{2}^{\mathbb{Z}}\right)$. Put

$$
G=C_{3}^{\mathbb{Z}} \rtimes_{\chi_{0} \circ \varphi_{1}} \lim _{\longleftarrow} C_{2}^{\mathbb{Z}} \text { and } \alpha=(\sigma, \tilde{\sigma}) .
$$

Identify $C_{3}^{\mathbb{Z}}$ and $\lim C_{2}^{\mathbb{Z}}$ with the obvious subgroups of $G$. Then $(G, \alpha)$ is a compact pair with $\operatorname{con}_{\leftrightarrow}(G)=C_{3}^{\mathbb{Z}}$ and $Z(G)=\operatorname{ker} \varphi_{1}$. Hence $Z(G) \operatorname{con}_{\leftrightarrow}(G)$ is a proper subgroup of $G$.

The group $G$ may also be described directly as an inverse limit, as follows. For each $n \in \mathbb{N}$ let $A_{n} \subset \mathbb{Z}$ be the support of $\varphi^{n}\left([\overline{1}]_{0}\right)$, where $\varphi: C_{2}^{\mathbb{Z}} \rightarrow C_{2}^{\mathbb{Z}}$ is defined by

$$
\varphi(g)(s)=g(s)-{ }_{2} g(s+1) .
$$

Define $\chi_{n}: C_{2}^{\mathbb{Z}} \rightarrow \operatorname{Aut}\left(C_{3}^{\mathbb{Z}}\right)$ to be the homomorphism that sends $[\overline{1}]_{s} \in C_{2}^{\mathbb{Z}}$ to

$$
\chi_{n}\left([\overline{1}]_{s}\right)(f)(r)=\left\{\begin{array}{ll}
\chi(f(r)), & \text { if } r-s \in A_{n} \\
f(r), & \text { otherwise }
\end{array}, \quad\left(f \in C_{3}^{\mathbb{Z}}\right)\right.
$$

and put $G_{n}=C_{3}^{\mathbb{Z}} \rtimes_{\chi_{n}} C_{2}^{\mathbb{Z}}$. Then calculation shows that $Z\left(G_{n}\right)$ is the subgroup of $C_{2}^{\mathbb{Z}}$ consisting of $2^{n}$-periodic elements. Let $\varphi_{n+1, n}: G_{n+1} \rightarrow G_{n}$ be defined at $(f, g)$ in $C_{3}^{\mathbb{Z}} \rtimes_{\chi_{n}} C_{2}^{\mathbb{Z}}$ by

$$
\varphi_{n+1, n}(f, g)=(f, \phi(g)) \text {. }
$$


The definition of the set $A$ in (20) implies that $\varphi_{n+1, n}$ is a homomorphism. Composing produces $\varphi_{m, n}: G_{m} \rightarrow G_{n}(m \geq n)$ and an inverse system $\left(G_{n}, \varphi_{m, n}\right)$. Then $G \cong \lim \left(G_{n}, \varphi_{m, n}\right)$.

\section{Topologically Transitive Automorphisms}

In his work on simple subgroups of automorphism groups of trees, 27, J. Tits introduced a property that he called $(\mathrm{P})$. This property, which is defined in terms of the action on the tree, has been important in subsequent papers on automorphism groups of trees, see [3, Section 3], and has been extended to groups of automorphisms of complexes in 9 . One of the important consequences of this property is found in [27, Lemme 4.3] and the next proposition is an abstract version of this result. In Tits' formulation of his Lemme 4.3, property $(\mathrm{P})$ asserts that the pair $\operatorname{nub}(\alpha), \alpha)$ is isomorphic to a shift over an infinite group. The hypothesis of the proposition is therefore considerably weaker.

Proposition 7.1. Let $(G, \alpha)$ be a compact pair with $\alpha$ topologically transitive. Then for every $k \in \mathbb{Z} \backslash\{0\}$ the map $\eta_{k}: x \mapsto x^{-1} \alpha^{k}(x)$ is surjective.

Proof. The proof is in three steps, the first of which is the case when $(G, \alpha)$ is a shift. The argument here is essentially the same as that in [27, Lemme 4.3]. Suppose that $(G, \alpha) \cong\left(F^{\mathbb{Z}}, \sigma\right)$ and let $f \in F^{\mathbb{Z}}$. Assume, without loss of generality, that $k>0$ and define $x \in F^{\mathbb{Z}}$ by $x(n)=1_{F}$ if $n \in\{0,1, \ldots, k-1\}$ and then define $x$ recursively by $x(n)=x(n+k) f(n)^{-1}$ if $n<0$ and $x(n)=x(n-k) f(n-k)$ if $n \geq k$. Then $f=\eta_{k}(x)$.

Next suppose that $S \leq G$ is normal, $\alpha$-stable, closed and such that $\left(G / S,\left.\alpha\right|^{G / S}\right)$ is isomorphic to a shift. Suppose also that the map $\left.\eta_{k}\right|_{S}:\left.x \mapsto x^{-1} \alpha^{k}\right|_{S}(x)$ is surjective on $S$. Let $f \in G$. Then, by the first paragraph, there is $x \in G$ such that $f=x^{-1} \alpha^{k}(x)$ modulo $S$. Hence $x f \alpha^{k}(x)^{-1}$ belongs to $S$ and, by assumption, there is $y \in S$ such that $x f \alpha^{k}(x)^{-1}=y^{-1} \alpha^{k}(y)$. Then $f=(y x)^{-1} \alpha^{k}(y x)=\eta_{k}(y x)$. An induction argument now shows that $\eta_{k}$ is surjective whenever $G$ has a composition series as in (12), that is, whenever $(G, \alpha)$ has finite depth.

Finally, suppose that $(G, \alpha)$ is topologically transitive and let $V$ be an open normal subgroup of $G$. Define $K_{0}=\bigcap_{k \in \mathbb{Z}} \alpha^{k}(V)$, so that $G / K_{0}$ has finite depth. Then $\alpha$ corestricts to an automorphism of $G / K_{0}$ and it follows by the previous paragraph that the map $x \mapsto x^{-1} \alpha^{k}(x)$ is surjective on $G$ modulo $K_{0}$. In particular, the map $\mathfrak{q}_{V} \circ \eta_{k}$ is surjective on $G / V$, where $\mathfrak{q}_{V}$ denotes the quotient map $G \rightarrow G / V$. Since this holds for every compact open subgroup $V$, it follows that $\eta_{k}(G)$ is dense in $G$. On the other hand, $\eta_{k}$ is continuous and $G$ is compact, so that $\eta_{k}(G)$ is closed. Therefore $\eta_{k}$ is surjective.

The map $\eta_{k}$ is generally not injective because there may be $k$-periodic elements of $G$, that is, elements $x$ satisfying $\alpha^{k}(x)=x$. This occurs, for example, for $\left(F^{\mathbb{Z}}, \sigma\right)$, where the set of periodic elements is dense. Moreover, it is shown in 25. Theorem 5.7] that, if $G$ is abelian and $(G, \alpha)$ has finite depth, then the set of points periodic for $\alpha$ is dense in $G$. The set of periodic points may fail to be dense if $(G, \alpha)$ does not have finite depth, as seen in [25, Examples 5.6]. For the case described in Example [5.11, the argument from [25] shows that the group $\lim G_{n}$ has no non-trivial $2^{j}$-periodic points for $\tilde{\sigma}$.

The final result shows that an element of a, not necessarily compact, group that is invariant modulo $\operatorname{nub}(\alpha)$ is equal modulo $\operatorname{nub}(\alpha)$ to an invariant element. 
Proposition 7.2. Let $G$ be a totally disconnected, locally compact group and let $\alpha \in \operatorname{Aut}(G)$. Suppose that $x \in G$ satisfies $\alpha(x \operatorname{nub}(\alpha))=x \operatorname{nub}(\alpha)$. Then there is $y \in x \operatorname{nub}(\alpha))$ such that $\alpha(y)=y$.

Proof. Let $h \in \operatorname{nub}(\alpha)$ be such that $\alpha(x)=x h$. Then for each $g \in \operatorname{nub}(\alpha)$ we have $\alpha(x g)=x h \alpha(g)$. Choose $g$ such that $g \alpha\left(g^{-1}\right)=h$ and put $y=x g$.

\section{REFERENCES}

[1] N. Aoki, Dense orbits of automorphisms and compactness of groups, Topology Appl. 20 (1985), 1-15.

[2] U. Baumgartner and G. A. Willis, Contraction groups and scales of automorphisms of totally disconnected locally compact groups, Israel J. Math. 142 (2004), 221-248.

[3] P.-E. Caprace and T. de Medts, Simple locally compact groups acting on trees and their germs of automorphisms, arXiv:1002.0220

[4] S. G. Dani, N. Shah, and G. A. Willis, Locally compact groups with dense orbits under $\mathbb{Z}^{d}$ actions by automorphisms, Ergodic Theory \& Dynamical Systems, 26 (2006), 1443-1465.

[5] van Dantzig, ???

[6] F. Fagnani, Some results on the classification of expansive automorphisms of compact abelian groups, Ergod. Th. \& Dynam. Sys., 16 (1996), 45-50.

[7] H. Glöckner, Contraction groups for tidy automorphisms of totally disconnected groups, Glasgow Math. J., 47 (2005), 329-333.

[8] H. Glöckner and G. A. Willis, Classification of the simple factors appearing in composition series of totally disconnected contraction groups, J. Reine Angew. Math. 643 (2010), 141-169.

[9] F. Haglund and F. Paulin, Simplicité de groupes d'automorphismes d'espaces à courbure négative, The Epstein birthday schrift, 181-48 (electronic), Geom. Topol. Monogr., 1, Geom. Topol. Publ., Coventry, (1998).

[10] P. R. Halmos, Lectures on Ergodic Theory, Publ. Math. Soc. Japan, Tokyo (1956).

[11] W. Hazod and E. Siebert, Automorphisms on a Lie group contracting modulo a compact subgroup and applications to semistable convolution semigroups, J. Theor. Probab., 1 (1988), $211-225$.

[12] E. Hewitt and K. A. Ross, Abstract Harmonic Analysis I, Grund. Math. Wiss. Bd 115, Springer-Verlag, Berlin-Göttingen-Heidelberg 1963.

[13] W. Jaworski, On contraction groups of automorphisms of totally disconnected locally compact groups, Israel J. Math., 172 (2009), 1-8.

[14] W. Jaworski, Contraction groups, ergodicity, and distal properties of automorphisms of compact groups, preprint.

[15] W. Jaworski, J. M. Rosenblatt and G.A. Willis, Concentration functions in locally compact groups, Math. Annalen 305(1996), 673-691.

[16] R. Kaufman and M. Rajagopalan, On automorphisms of a locally compact group, Michigan Math. J. 13 (1966), 373-374.

[17] B. Kitchens, Expansive dynamics of zero-dimensional groups, Ergod. Th. \& Dynam. Sys., 7 (1987), 249-261.

[18] B. Kitchens and K. Schmidt, Automorphisms of compact groups, Ergod. Th. \& Dynam. Sys. 9 (1989), 691-735.

[19] B. Kitchens and K. Schmidt, Isomorphism rigidity of irreducible $\mathbb{Z}^{d}$-actions, Inventiones mathematicae 142 (2000), 559-577.

[20] S. Lang, Algebra, Graduate Texts in Mathematics (Revised Third Edition), Springer-Verlag, New York, (2002).

[21] D. Lind and K. Schmidt, Homoclinic points of algebraic $\mathbb{Z}^{d}$-actions, Journal of the American Mathematical Society, 12 (1999), 953-980.

[22] D. Montgomery and L. Zippin, Topological Transformation Groups, Interscience Publishers, New York-London 1955.

[23] C. C. Moore, The Mautner phenomenon for general unitary representations, Pac. J. Math., 86 (1980), 155-169.

[24] W. H. Previts and T.-S. Wu. Dense orbits and compactness of groups, Bull. Austral. Math. Soc., 68(1):155-159, 2003. 
[25] K. Schmidt, Dynamical Systems of Algebraic Origin, Progress in Mathematics 128, Birkhäuser, 1995.

[26] E. Siebert, Contractive automorphisms on locally compact groups, Math. Zeitschrift, 191 (1986), 73-90.

[27] J. Tits, Sur le groupe d'automorphismes d'un arbre, In A. Haeflinger and R. Narisimhan (editors), Essays on topology and related topics (Memoires dédiés à Georges de Rham, pp 188-211, Springer Verlag, New York, 1970.

[28] G. A. Willis, The structure of totally disconnected, locally compact groups, Math. Ann. 300 (1994), 341-363.

[29] J. S. P. Wang, The Mautner phenomenon for p-adic Lie groups, Math. Z., 185 (1984), 403412.

[30] G. A. Willis, Totally disconnected groups and proofs of conjectures of Hofmann and Mukherjea, Bull. Australian Math. Soc. 51(1995), 489-494.

[31] G. A. Willis, Further properties of the scale function on a totally disconnected group, J. Algebra 237 (2001), 142-164.

[32] G. A. Willis, Tidy subgroups for commuting automorphisms of totally disconnected groups: an analogue of simultaneous triangularisation of matrices, New York J. Math. 10(2004), 1-35. (Available at http://nyjm.albany.edu:8000/j/2004/Vol10.htm)

[33] G. A. Willis, A canonical form for automorphisms of totally disconnected locally compact groups, in Random walks and geometry, Walter de Gruyter, Berlin, (2004), 295-316.

Department of Mathematics, University of Newcastle, Callaghan, NSW 2308, AusTRALIA

E-mail address: george.willis@newcastle.edu.au 